Accepted to AJ

Preprint typeset using LATEX style AASTeX6 v. 1.0

\title{
ZODIACAL EXOPLANETS IN TIME (ZEIT) IV: SEVEN TRANSITING PLANETS IN THE PRAESEPE CLUSTER
}

\begin{abstract}
Andrew W. Mann ${ }^{1,2}$, Eric Gaidos ${ }^{3}$, Andrew Vanderburg ${ }^{4,5}$, Aaron C. Rizzuto ${ }^{1}$, Megan Ansdell ${ }^{6}$, Jennifer Vanessa Medina ${ }^{1,7}$, Gregory N. Mace $^{1}$, Adam L. Kraus ${ }^{1}$, Kimberly R. Sokal ${ }^{1}$
\end{abstract}

\footnotetext{
${ }^{1}$ Department of Astronomy, The University of Texas at Austin, Austin, TX 78712, USA

${ }^{2}$ Hubble Fellow

${ }^{3}$ Department of Geology and Geophysics, University of Hawaii at Manoa, Honolulu, HI 96822, USA

${ }^{4}$ Harvard-Smithsonian Center for Astrophysics, Cambridge, Massachusetts 02138, USA

${ }^{5}$ NSF Graduate Research Fellow

${ }^{6}$ Institute for Astronomy, University of Hawaii at Manoa, Honolulu, HI 96822, USA

${ }^{7}$ TAURUS Scholar
}

\begin{abstract}
Open clusters and young stellar associations are attractive sites to search for planets and to test theories of planet formation, migration, and evolution. We present our search for, and characterization of, transiting planets in the 800 Myr old Praesepe (Beehive, M44) Cluster from K2 light curves. We identify seven planet candidates, six of which we statistically validate to be real planets, the last of which requires more data. For each host star we obtain high-resolution NIR spectra to measure its projected rotational broadening and radial velocity, the latter of which we use to confirm cluster membership. We combine low-resolution spectra with the known cluster distance and metallicity to provide precise temperatures, masses, radii, and luminosities for the host stars. Combining our measurements of rotational broadening, rotation periods, and our derived stellar radii, we show that all planetary orbits are consistent with alignment to their host star's rotation. We fit the K2 light curves, including priors on stellar density to put constraints on the planetary eccentricities, all of which are consistent with zero. The difference between the number of planets found in Praesepe and Hyades (8 planets, $\simeq 800 \mathrm{Myr}$ ) and a similar dataset for Pleiades (0 planets, $\simeq 125 \mathrm{Myr}$ ) suggests a trend with age, but may be due to incompleteness of current search pipelines for younger, faster-rotating stars. We see increasing evidence that some planets continue to lose atmosphere past $800 \mathrm{Myr}$, as now two planets at this age have radii significantly larger than their older counterparts from Kepler.

Keywords: planets and satellites: dynamical evolution and stability — planets and satellites: detection - stars: fundamental parameters - stars: low-mass - stars: planetary systems - the Galaxy: open clusters and associations: individual
\end{abstract}

\section{INTRODUCTION}

Stellar clusters are unique sites to test theories of planet formation and evolution. Because they consist of chemically homogenous (or nearly homogeneous, Liu et al. 2016) stellar populations, planets in clusters facilitate statistical studies of exoplanet properties (e.g., occurrence, period, size) while controlling for changes due to host star metallicity (e.g., Buchhave et al. 2012; Neves et al. 2013; Mann et al. 2013b). Common and well-measured age, metallicity, and distance help yield more precise determinations of stellar parameters for cluster members than is generally possible for field stars, often providing a proportional improvement in planet parameters. The comparatively well constrained ages available for clusters compared to field stars (e.g., Schneider et al. 2014;
Cargile et al. 2014; Dahm 2015) also facilitate studies of planetary evolution. To this end, young $(<1 \mathrm{Gyr})$ clusters are particularly useful because planetary systems undergo the most change in the first few hundred megayears (e.g., Adams \& Laughlin 2006; Raymond et al. 2009). The Kepler mission (Borucki et al. 2010) has found thousands of (candidate) planets (e.g., Mullally et al. 2015). However, target stars in the Kepler-prime mission are generally older than $1 \mathrm{Gyr}$, and most have poorly constrained ages (Batalha et al. 2010; Walkowicz \& Basri 2013; Silva Aguirre et al. 2015), making them less useful for evolutionary studies. Planets in young stellar clusters could fill this gap.

Because of their scientific value, open clusters have long been targeted for exoplanet searches (e.g., Cochran 
et al. 2002; Mochejska et al. 2002; Aigrain et al. 2007; van Saders \& Gaudi 2011). Despite numerous surveys, only a handful of planets in open clusters were discovered prior to Kepler (e.g., Lovis \& Mayor 2007; Quinn et al. 2012), and none were significantly smaller or less massive than Jupiter. These early searches were generally only sensitive only to Jovian planets on close orbits, which are intrinsically rare (e.g., Johnson et al. 2010; Gaidos et al. 2013; Fressin et al. 2013). Most nearby clusters $(<200 \mathrm{pc})$ are younger than $1 \mathrm{Gyr}$, and thus their members are noisier (in terms of radial velocity (RV) and photometric variation) than their older counterparts (Paulson et al. 2004; Reiners et al. 2010; Crockett et al. 2012), which complicates the detection and characterization of any planetary signal. Because of brightness limitations, studies of more distant, older clusters were limited to brighter F-, G-, and early-K-type members, which removes $>70 \%$ of the potential targets and makes detecting even a few planets unlikely. Though recent improvements in sensitivity may enable RV surveys to detect down to Neptune-mass objects on close orbits in the coming years (Malavolta et al. 2016; Quinn et al. 2014).

The Kepler spacecraft can detect the much more common Earth-to-Neptune-size planets (Jenkins et al. 2010), which has enabled the discovery of two planets smaller than Neptune in the open cluster NGC6811 (Meibom et al. 2013). However, NGC6811 is $\sim 1$ Gyr old, and resides at a distance of $1100 \mathrm{pc}$, which provides limited temporal information and makes follow-up difficult. The Kepler-prime field contains no open clusters clusters that are significantly younger or closer than NGC6811 within which to search for planets.

The repurposed Kepler mission K2, (Howell et al. 2014) provides a unique opportunity to revisit open clusters for planet searches. So far K2 has observed Praesepe, Hyades, M35, and Pleiades, the young star-forming regions Upper Scorpius and $\rho$ Ophucus, and older clusters M67 and Ruprecht 147. K2 observations of TaurusAuriga and additional visits to Hyades, Upper Scorpius, and Praesepe are planned for future campaigns. They span ages of $\simeq 2$ to $800 \mathrm{Myr}$, supplying an unparalleled dataset to explore planetary (and stellar) evolution. These groups are all sufficiently close $(<200 \mathrm{pc})$ to search for planets around the more numerous M-dwarf members. Furthermore, because K2 is sensitive to super-Earth and Neptune-size planets (Dressing \& Charbonneau 2013; Petigura et al. 2013; Gaidos et al. 2016) around most target stars (e.g. Vanderburg et al. 2016; Crossfield et al. 2016) the expected planet yield is much higher than earlier surveys.

To take full advantage of the $K 2$ data set, we launched the Zodiacal Exoplanets in Time (ZEIT) survey. Our aims are to identify, characterize, and explore the statis- tical properties of planets in nearby young clusters and star-forming regions utilizing both $K 2$ light curves and a suite of ground-based instruments for follow-up. Our long-term goal is to gain a better understanding of the evolution of planets from infancy $(<10 \mathrm{Myr})$ to maturity $(>1 \mathrm{Gyr})$, including changes in their physical properties, dynamics, and atmospheres. Thus far, we have identified two planets, one in the $\simeq 800$ Myr old Hyades cluster (Mann et al. 2016a), and one in the $\simeq 11$ Myr old Upper Scorpius OB association (Mann et al. 2016b) (see also, David et al. 2016b,a, who independently discovered both systems). Our search of the $\simeq 125 \mathrm{Myr}$ old Pleiades cluster data turned up only a single planet, which is more likely to be a young field star with Pleiades-like kinematics than a true cluster member (Gaidos et al. 2017). These planets represent important age benchmarks, and can be used to improve our understanding of planetary evolution, but the inclusion of only two planets is insufficient for statistical work.

Here we present our search for, and characterization of, planets in the Praesepe cluster (also known as the Beehive cluster or M44). In total, we found seven planet candidates, which we follow-up with ground-based spectroscopy and adaptive optics imaging of the host stars (Section 2). We combine these data with literature photometry and astrometry to constrain the properties (mass, radius, etc.) of each host star and confirm their membership to Praesepe (Section 3). In Section 4 we describe our fit of the transit light curves in order to constrain planetary properties, including eccentricity. We use publicly available software to assess the false-positive probability of each system in Section 5, with which we confirm the planetary nature of six out of seven planet candidates. We conclude in Section 6 with a discussion of the dynamical state, frequency, and size of the Praesepe and Hyades planets when compared to significantly older systems.

\section{OBSERVATIONS AND DATA REDUCTION}

\subsection{K2 Observations and Transit Identification}

From 2015 April 27 to 2015 October 31 (Campaign 5), K2 observed $\sim 900$ known members of the Praesepe cluster. Owing to the loss of two reaction wheels, the Kepler spacecraft drifts on < day timescales (Van Cleve et al. 2016). To correct the pointing, Kepler's thrusters fire every $\sim 6$ hours. However, during the drift and subsequent thruster fire, stellar images will drift with respect to the detector. Combined with variations in the pixel sensitivity, this drift generates changes in total measured flux from a given star as a function of centroid position.

Multiple methods have been implemented to mitigate or remove noise from K2 drift. We utilized both 'K2SFF' (Vanderburg \& Johnson 2014) and 'K2SC' (Aigrain et al. 
2016) corrected light curves for all targets. K2SFF curves were corrected for noise due to telescope drift by correlating flux measurements with the spacecraft's pointing. K2SC curves were derived using Gaussian Process regression to model changes that depend on the target's position (flat field variability) and time (stellar variability) simultaneously. We also extracted our own light curves, following the method of Vanderburg \& Johnson (2014), but including a simultaneous fit for stellar variability with a lower (1 day) cutoff on the stellar rotation period than allowed by Vanderburg \& Johnson (2014). Some Praesepe-age M-dwarfs will have rotation periods shorter than this (Douglas et al. 2014), but K2 long-cadence observations yield too few data points for significantly shorter period cutoffs, and fitting out variation on $\sim$ hour timescales runs the risk of removing or altering long-duration transits.

We downloaded (or extracted from the pixel data) light curves for all Praesepe members given in Kraus \& Hillenbrand (2007) observed by K2 from the Barbara A. Mikulski Archive for Space Telescopes (MAST). We ran a box least-squares (Kovács et al. 2002) search for transits on each light curve after correcting for stellar variability. Additional details on our search method are given in Gaidos et al. (2017). Eclipsing binaries were identified visually for separate analysis (A. L. Kraus et al., in preparation). Other artifacts from poorly corrected stellar variability, flares, or red noise are flagged by identifying changes in the transit shape and depth with time, comparing the planet candidate's orbital period to the stellar rotation period, and examining the transit by eye (though no candidates were rejected through visual examination alone). We used our own curves to verify that candidate signals were not artifacts of the reduction process. If a planet was identified in K2SFF or K2SC light curves and not in our curves we re-extracted the relevant light curve, manually locking the stellar rotation correction to the value derived from a Lomb-Scargle periodogram, and removing outliers manually. It is infeasible to repeat this process for all target stars, but it is simple to do on the few with potential signals. In this way all candidate signals were eventually identified with our own light curve.

In total, seven planet candidates survived our vetting process. Four of these planets have been previously identified by earlier analyses of the K2 data (Libralato et al. 2016; Barros et al. 2016; Pope et al. 2016), two of which were recognized as orbiting Praesepe members (Libralato et al. 2016), and one of which (K2-95) was characterized in detailed and confirmed to be planetary by Obermeier et al. (2016).

For each of the candidates, we extracted a new light curve after the transit was identified. Re-extraction was done because corrections for K2 pointing drift and stellar variability may incorrectly fit out or otherwise negatively affect the transit (Grunblatt et al. 2016). Once the transit is identified, we can include this in the fit to eliminate or mitigate systematic errors introduced this way. Following Becker et al. (2015) and Mann et al. (2016a), we simultaneously fit for low frequency variations from stellar activity, Kepler flat field (drift), and the transits of each system using a least-squares minimization. Both stellar variability and the effect of errors in detector response were modeled as splines as a function of time and centroid position with breakpoints every 0.2 days and $0.4^{\prime \prime}$, respectively. We used the re-extracted and flattened light curves for measuring transit properties (Section 4), but used light curves with only basic processing (flat field/drift correction) for measuring stellar rotation periods.

\subsection{Optical Spectra from SNIFS}

On 2016 January 17 (UT), we obtained an optical spectrum of each target with the SuperNova Integral Field Spectrograph (SNIFS, Aldering et al. 2002; Lantz et al. 2004) on the University of Hawai'i $2.2 \mathrm{~m}$ telescope on Maunakea. SNIFS covers $3200-9700 \AA$ simultaneously with a resolution of $R \simeq 700$ and $\mathrm{R} \simeq 1000$ in the blue $(3200$ $5200 \AA)$ and red (5100-9700 $\AA$ ) channels, respectively. Exposure times varied from 60 to 1800 s, providing a typical $\mathrm{S} / \mathrm{N}=90$ per resolving element near $6500 \AA$. ThAr arcs were taken before or after each observation, which helps to extract the spectrum and improve the wavelength solution. Bias, flat, dark correction and cosmic-ray rejection, construction of the data cubes, and extraction of the one-dimensional spectrum are described in detail in Aldering et al. (2002). We observed spectrophotometric standards throughout the night, which were used in conjunction with a model of the atmospheric absorption above Maunakea to telluric correct and flux calibrate the spectrum. More details on our observing and reduction methods can be found in Mann et al. (2015).

\subsection{Near-infrared Spectra with SpeX}

During the nights of 2016 January 29, February 21, or March 5 (UT), we obtained a spectrum of each target with the near-infrared (NIR) spectrograph SpeX, mounted on the Infrared Telescope Facility on Maunakea. Observations were taken in cross-dispersed (SXD) mode with the $0.3^{\prime \prime}$ slit, yielding a resolution of $\simeq 2000$ with complete coverage from $0.7-2.5 \mu \mathrm{m}$. Each target was placed on two positions (A and B) on opposite ends of the slit. After each integration, the object was nodded following an ABBA pattern. Image differences $(A-B)$ were used to subtract emission from the atmosphere. Integration times varied based on the brightness of the target, but all were capped at 120s per exposure to mitigate atmospheric variations. For fainter targets, more ABBA 
sequences were taken until the desired $\mathrm{S} / \mathrm{N}$ was reached. For all targets we obtained an $\mathrm{S} / \mathrm{N}$ per resolving element of $>60$ in the center of the $H$ and $K$ bands. Internal flat and arc lamps and A0V standards were observed for each target at a similar airmass and sky position as the target. Individual spectra were reduced, extracted, and stacked using the SpeXTool package (Cushing et al. 2004). Telluric correction and flux calibration were applied using the A0V standard and the xtellcor package (Vacca et al. 2003).

\subsection{High-resolution Spectra with IGRINS}

We observed each of the seven planet hosts during the nights of 2016 February 24 or April 21 (UT) with the Immersion Grating Infrared Spectrometer (IGRINS, Park et al. 2014; Mace et al. 2016) on the 2.7m Harlan J. Smith telescope located at McDonald Observatory. IGRINS uses a silicon immersion grating (Yuk et al. $2010)$ to achieve high resolving power $(R \simeq 45,000)$ and simultaneous coverage of both $H$ and $K$ bands (1.48$2.48 \mu \mathrm{m}$ ) on two separate Hawaii-2RG detectors. IGRINS is stable enough to achieve RV precision of $\lesssim 40 \mathrm{~m} \mathrm{~s}^{-1}$ by using telluric lines for wavelength calibration.

Due to a higher false-positive probability (see Section 5) and a transit shape consistent with a grazing eclipsing binary, we obtained three additional epochs of one target (EPIC 211901114). These were taken on 2016 October 10, 11, and 12 (UT) with IGRINS on the Discovery Channel Telescope.

All observations were taken following commonly used strategies for point-source observations with IGRINS. To briefly summarize, each target was placed at two positions along the slit (A and B), taking an exposure at each position in an ABBA pattern as with the SpeX observations. Exposure times varied based on the target's $K_{S}$ magnitude, but were capped at 600s to avoid saturation of sky emission lines. For the faintest targets, additional ABBA sequences were taken until the required $\mathrm{S} / \mathrm{N}$ was achieved. To help remove telluric lines, A0V standards were observed following the same pattern. Enough A0V standards were taken to ensure there was at least one standard taken within 0.1 airmasses and 1 hour (of time) of every target.

The IGRINS spectra were reduced using version 2.1 of the publicly available IGRINS pipeline package ${ }^{1}$ (Lee 2015), which includes flat-fielding, background removal, order extraction, distortion correction, wavelength calibration, and basic telluric correction using the A0V standards and an A star atmospheric model. Spectra without telluric corrections applied were preserved and used to improve the wavelength solution and provide a

${ }^{1}$ https://github.com/igrins/plp zero-point for the RVs.

\subsection{Adaptive Optics Imaging and Aperture Masking}

During the nights of 2016 March 19, and March 22, (UT), we observed four of the seven planet hosts (K2-100, $\mathrm{K} 2-101, \mathrm{~K} 2-102$, and K2-103) using natural guide star (NGS) adaptive optics (AO) imaging (Wizinowich et al. 2000) and non-redundant aperture masking (NRM). The three other targets (K2-95, K2-104, and EPIC 211901114) are too faint for NGS, and the Keck 2 laser was not operational during these two nights.

All observations were taken with the facility imager, NIRC2, on Keck II atop Maunakea. Vertical angle mode was used for both imaging and NRM observations, always utilizing the smallest pixel scale (9.952 \pm 0.002 mas $/$ pix $)$. Imaging was taken with the $K^{\prime}$ or $K_{c}$ (for K2-100) filter and masking with the nine-hole mask. After AO loops closed on each target, we took four to eight images, adjusting coadds and integration time based on the brightness of the target. For NRM, we took six interferograms, each with an integration of time of $20 \mathrm{~s}$ and a single coadd.

Data reduction and analysis was done following Kraus et al. (2016). To summarize, each frame was linearized and corrected for distortion using the NIRC2 solution from Yelda et al. (2010), then dark and flat corrected using calibration data taken the same night. We interpolated over "dead" and "hot" pixels, which were identified from superflats and superdarks built from data spanning 2006 to 2013. Pixels with flux levels $>10 \sigma$ above the median of the eight adjacent pixels (cosmic rays) were replaced with the median. We searched for faint and wide companions in the AO imaged by first subtracting an azimuthal median PSF model. Close-in companions were identified by first constructing and subtracting a best-fit PSF of another (single-star) taken on one of the two observing nights. All images of a given target were stacked, and searched for companions using 40 mas radius apertures centered on each pixel. Detection limits were determined from the standard deviation of the flux among all apertures.

Reduction of masking observations follows the appendix of Kraus et al. (2008). To remove systematics, the observation of each target was paired with a calibration observation of another nearby member of Praesepe or known single-star calibrator taken from Hartkopf et al. (2001). Binary system profiles were then fit to the closure phase to produce detection limits. More details on the reduction of masking data can be found in Kraus et al. (2008) and Kraus et al. (2016).

Detection limits (in terms of contrast ratio) as a function of separation constructed from the combination of masking and imaging for the four targets observed are shown in Figure 1. Owing to the edges of the detector the 


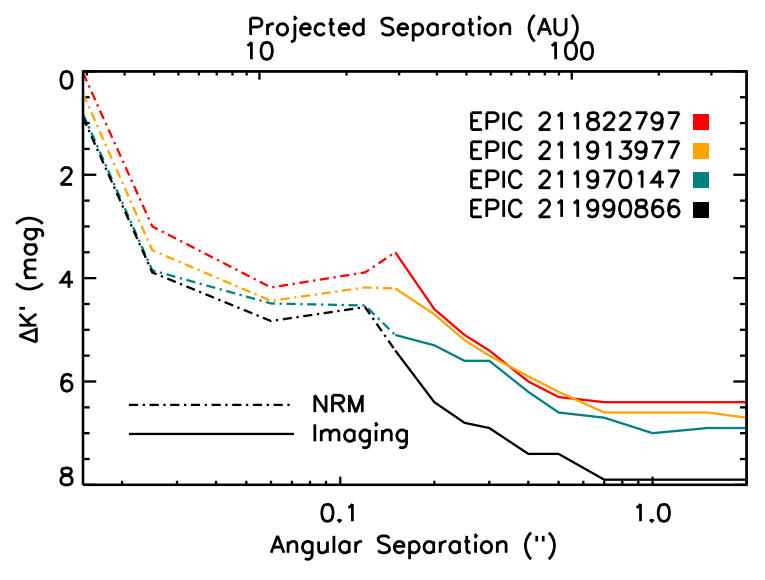

Figure 1. Detection limits $(5 \sigma)$ as a function of separation for the four targets with AO imaging and NRM interferometry. The top axis shows the separation in AU assuming a distance of $182 \mathrm{pc}$. The region probed by non-redundant aperture masking is marked with a dotted-dashed line, while the region probed by imaging is solid. The contrast limits are approximately flat for separations $>3^{\prime \prime}$, but due to finite chip size are incomplete as a function of azimuthal angle.

azimuthal coverage is not complete past $\simeq 3^{\prime \prime}$ depending on where the object was placed on the detector. Only one target had a significant detection; the images of K2100 show a faint $\left(\Delta K^{\prime}=5.830 \pm 0.010\right)$ companion at a separation of $1017.7 \pm 1.6$ mas and a position angle of $98.623 \pm 0.090$ degrees. The companion is close enough to land in the selected $K 2$ aperture (pixel size $=3.98^{\prime \prime}$ ). However, for any physical $K_{P}-K$ (i.e., a star that is sufficiently blue and faint would land outside the galaxy) the fainter star is too faint to account for, or significantly dilute the transit depth of K2-100b, and was therefore ignored.

\section{STELLAR PARAMETERS}

Common parameters for all targets: for each of the seven planet hosts, we assume a common $[\mathrm{Fe} / \mathrm{H}]$, reddening, distance, and age. We adopt $[\mathrm{Fe} / \mathrm{H}]=0.14 \pm 0.04$, which encompasses measurements from the literature derived from high-resolution spectra of FGK stars (Boesgaard et al. 2013; Yang et al. 2015; Netopil et al. 2016) and any chemical inhomogeneities as observed in similar clusters ( $\simeq 0.02$ dex in Hyades, Liu et al. 2016). We adopt a reddening of $E(B-V)=0.027 \pm 0.004 \mathrm{mag}$ measured by Taylor (2006). Because the cluster is relatively compact ( $1 \sigma \simeq 2 \mathrm{pc}$ ), and cluster and circumstellar gas (and dust) have been dispersed, reddening variation between targets should be smaller than measurement uncertainties. van Leeuwen (2009) measure a Hipparcosbased distance of $181.5 \pm 6.0 \mathrm{pc}$ to the core of Praesepe, which we use for each of the individual stars. As with reddening, the distance error is large enough to account for the scatter in individual object distances due to the finite size of the cluster core. This distance is consistent with independent measurements for Praesepe (e.g., Gáspár et al. 2009). Main-sequence turnoff and isochrone fitting suggest an age for Praesepe and Hyades of 600-700 Myr (Perryman et al. 1998; Salaris et al. 2004), but accounting for the effects of rotation and revisions to the solar metallicity scale suggest an older age of $\simeq 800 \mathrm{Myr}$ (Brandt \& Huang 2015). For our analysis, we adopted the older age of $790 \pm 30$ Myr from Brandt \& Huang (2015). Global parameters are summarized in Table 1.

Table 1. Global Parameters

\begin{tabular}{lll}
\hline \hline \multicolumn{1}{c}{ Parameter } & Value & \multicolumn{1}{c}{ Source } \\
& & \\
\hline Age (Myr) & $790 \pm 30 \mathrm{Myr}$ & Brandt \& Huang (2015) \\
\hline & & Boesgaard et al. (2013), \\
{$[\mathrm{Fe} / \mathrm{H}]$} & $0.14 \pm 0.04$ & $\begin{array}{l}\text { Yang et al. (2015), } \\
\text { Netopil et al. (2016) }\end{array}$ \\
\hline $\mathrm{E}(\mathrm{B}-\mathrm{V})(\mathrm{mag})$ & $0.027 \pm 0.004$ & Taylor (2006) \\
Distance (pc) & $181.5 \pm 6.0$ & van Leeuwen (2009) \\
\hline
\end{tabular}

Radial Velocities: RVs were determined from the IGRINS data as explained in Mann et al. (2016a) and G. N. Mace et al. (in preparation). To briefly summarize, we used telluric lines to improve the wavelength solution and provide a fixed zero-point across all observations. We then cross-correlated each IGRINS spectra against 150-230 spectra of RV standards with similar spectral types to the target. The final assigned RV and error is the robust mean and standard error of the cross-correlation across all templates. For targets with multiple measurements, we used the weighted mean of the measurements. For absolute RVs, errors are limited by the zero-point error of $153 \mathrm{~m} \mathrm{~s}^{-1}$, which is due to limits on the RV precision of the templates.

Membership in Praesepe: all planet hosts are included in the Praesepe membership catalog of Kraus \& Hillenbrand (2007) with membership probabilities of $\geq 97 \%$. However, Kraus \& Hillenbrand (2007) calculations use only proper motions and photometry. RVs derived from our IGRINS spectra enable the calculation of more precise, three-dimensional probabilities. We first measured each target's photometric distance by comparing available optical and NIR photometry against the solar metallicity isochrones from Dotter et al. (2008). We drew position and proper motion information from UCAC4 (Zacharias et al. 2013) or SDSS (Ahn et al. 2012), where available. We then computed Galactic $U V W$ kinematics by combining these data with the RVs. Membership probabilities were calculated following the Bayesian framework of Rizzuto et al. (2011). We drew Praesepe $U V W$ kinematics from van Leeuwen (2009) and values 
for field stars from Malo et al. (2013). We selected a membership prior equal to the ratio of the number of stars in Praesepe to the number of field stars in the same region of the sky. To this end, we constructed a Praesepe CMD from APASS or SDSS $r-2$ MASS $K$ color of Praesepe members identified by Kraus \& Hillenbrand (2007). We considered all stars with $r<17$, within $8^{\circ}$ of the Praesepe core, and $5 \sigma$ of the Praesepe color-magnitude diagram (CMD); those not in the Kraus \& Hillenbrand (2007) catalog, we assigned as field stars and those in Kraus \& Hillenbrand (2007) we assigned as members, ignoring the individual membership probabilities for simplicity. The resulting Bayesian membership probabilities were $>99.9 \%$ for all seven planet hosts.

RVs have not been measured for the majority of cluster members (especially low-mass members), so instead of $U V W$ we show positions and proper motions of planet hosts and cluster members in Figure 2, and CMD positions in Figure 3. All planet hosts are consistent with the kinematics and position of the cluster, and all planet hosts have CMD positions consistent with the single-star cluster sequence.

Effective temperatures: we compared our dereddened spectra to a grid of BT-SETTL CIFIST stellar atmosphere models ${ }^{2}$ (Allard et al. 2012). For the four Mdwarfs in the sample, we mask out regions where models poorly reproduce observed spectra as detailed in Mann et al. (2013a), and for all stars we mask out regions of high telluric contamination. We included five nuisance parameters to deal with small errors in the wavelength and flux calibration of SNIFS and SpeX (see Gaidos et al. 2014; Mann et al. 2015, for more details). We estimated errors due to uncertainties in the reddening by repeating the fitting process over the range of $E(B-V)$ values $(0.027 \pm 0.004)$ and find the change in $T_{\text {eff }}$ is negligible compared to other errors.

Our model-fitting method reproduces M-dwarf temperatures measured from long-baseline optical interferometry (Boyajian et al. 2012b; Mann et al. 2013a). For the three warmer stars we tested our method using a similar sample FGK dwarfs with interferometric temperatures from (Boyajian et al. 2012a), which we also used to estimate the errors on our method and test for systematic offsets. Our final $T_{\text {eff }}$ values for the planet hosts are also consistent with those derived from color$T_{\text {eff }}$ relations from Mann et al. (2015) for the M-dwarfs and from Ramírez \& Meléndez (2005) and Pinsonneault et al. (2012) for FGK dwarfs.

We estimated $T_{\text {eff }}$ for other cluster members using available APASS, 2MASS, and Tycho-2 (Høg et al. 2000)

2 https://phoenix.ens-lyon.fr/Grids/BT-Settl/CIFIST2011
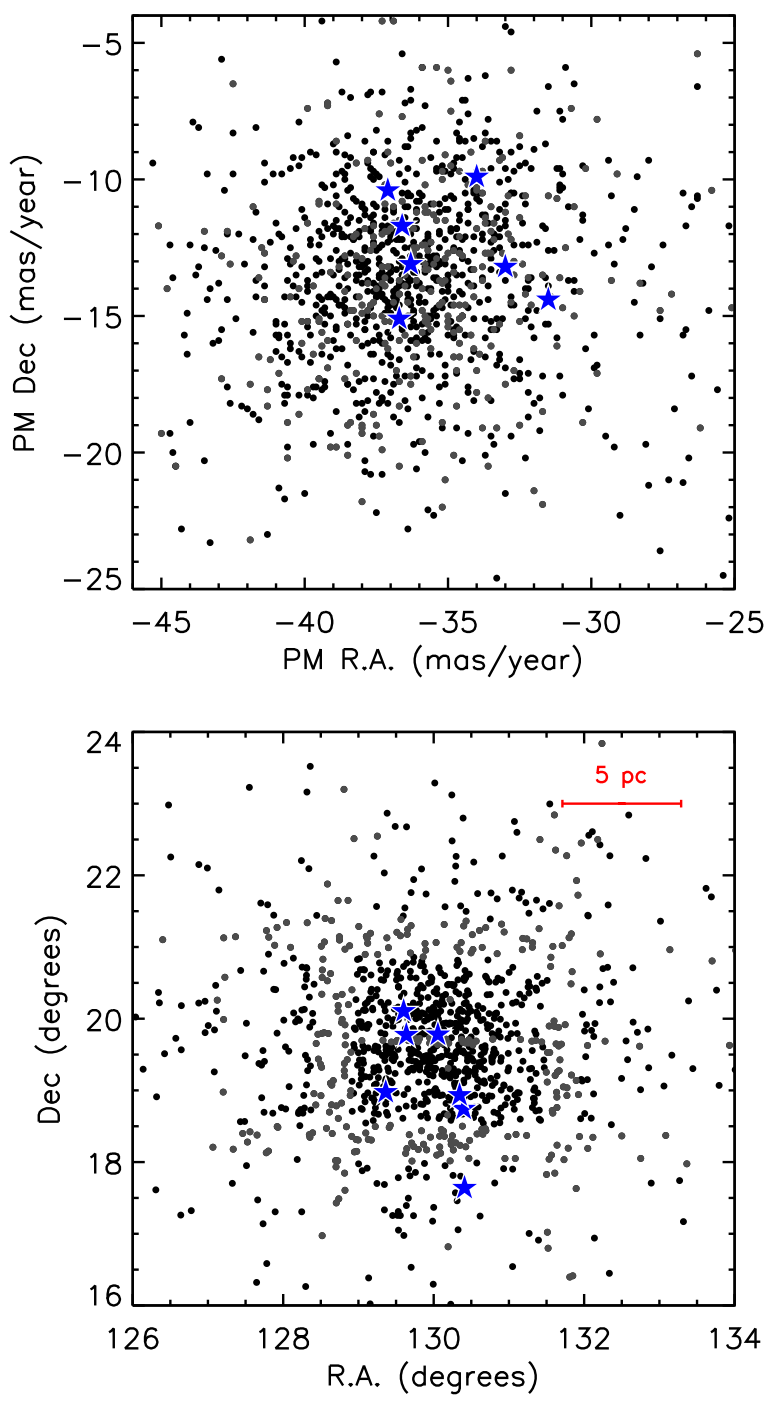

Figure 2. Proper motions (top) and positions (bottom) of likely Praesepe members from Kraus \& Hillenbrand (2007). An estimate of the (projected) physical scale is shown in the center plot based on the distance to the cluster. Targets not observed by $K 2$ are shown in gray. Planet hosts are shown as blue stars. Plot edges cut off some $(<5 \%)$ members to better show detail in the core.

photometry of likely members and the color- $T_{\text {eff }}$ relations from Mann et al. (2015) for the M-dwarfs and from Ramírez \& Meléndez (2005) and Pinsonneault et al. (2012) for FGK dwarfs. We show the resulting $T_{\text {eff }}$ values with luminosities (see below) for planet hosts and members in Figure 3.

Bolometric fluxes: we compiled well-calibrated photometry from the literature; $B V$ gri from the ninth data release of the AAVSO All-Sky Photometric Survey (APASS, Henden et al. 2012), $J H K_{S}$ from The Two Micron All Sky Survey (2MASS, Skrutskie et al. 2006), griz from the Sloan Digital Sky Survey (SDSS, Ahn et al. 2012), and $W 1 W 2 W 3$ photometry from the Wide-field Infrared Survey Explorer (WISE, Wright et al. 2010). We then 

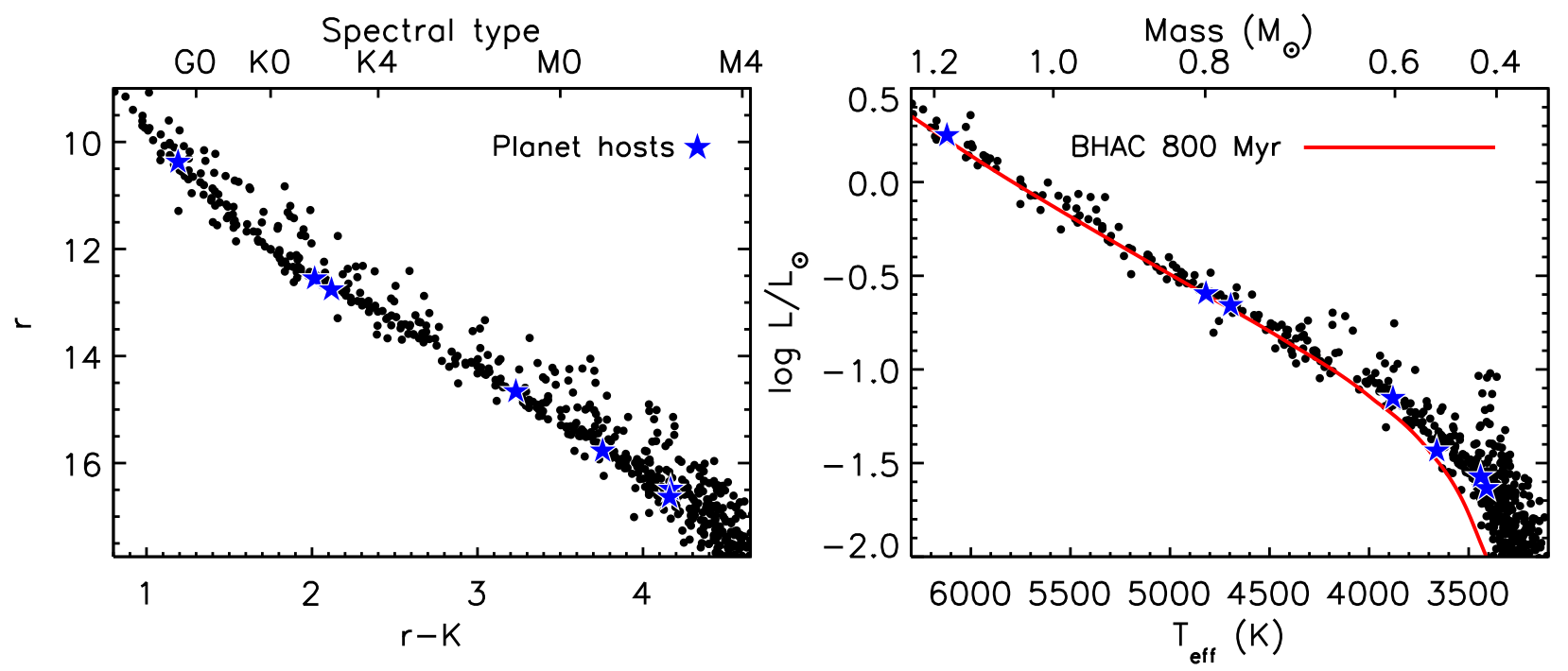

Figure 3. Color-magnitude (left) and H-R diagram (right) for planet hosts (blue stars) and likely Praesepe members (black points) from Kraus \& Hillenbrand (2007), with an 800 Myr isochrone from Baraffe et al. (2015) in red on the right panel. Approximate spectral types (left) and masses (right) are shown in the top axes. The bluest and reddest stars are cut from both plots to show more detail in the sequence near the planet hosts, and some stars are not shown in one or both plots due to a lack of sufficient reliable photometry.

scaled the (still reddened) NIR and optical spectrum to the archival photometry following the procedure from Mann et al. (2015), including filling in regions outside our observed spectra $(0.35-2.4 \mu \mathrm{m})$ and areas of high telluric absorption with an atmospheric model. To calculate the bolometric flux $\left(F_{\text {bol }}\right)$, we removed the effects of extinction/reddening from the combined and calibrated spectrum using the reddening law from Cardelli et al. (1989), and then integrated the spectrum over all wavelengths. As with $T_{\text {eff }}$, we repeated our routine using the range of possible $E(B-V)$ values, which had only a marginal effect on our overall $F_{\text {bol }}$ errors.

Stellar radius, luminosity, and mass: combining $F_{\mathrm{bol}}$, $T_{\text {eff }}$, and distance, we calculated stellar radii using the Stefan-Boltzman relation. We similarly computed luminosities from the distance and $F_{\text {bol }}$. We derived unreddened, synthetic $K$ and $V$ magnitudes from our calibrated spectra using the filter profiles and zero-points from Cohen et al. (2003) and Mann \& von Braun (2015), which we converted to absolute magnitudes using the cluster distance. With these, we estimated host star masses using the the semi-empirical mass $-M_{K}$ relation from Mann et al. (2015), which reproduces the massradius relation from low-mass eclipsing binaries (Feiden \& Chaboyer 2012), and mass-luminosity relation from astrometric binaries (Delfosse et al. 2000). For the warmer stars, we use the empirical mass $-M_{V}$ relation from Henry \& McCarthy (1993). As a check, we also derive masses by interpolating $M_{J}, M_{H}$, and $M_{K}$ onto $800 \mathrm{Myr}$ stellar isochrones from Baraffe et al. (2015). The model-based masses are all within $1 \sigma$ of those estimated above, but give errors that may be unrealistically small due to systematic errors in the underlying models, so we adopt the more empirical values.

Luminosities for the cluster population were estimated using the cluster distance and $r$-band bolometric corrections from Mann et al. (2015) or $V$-band bolometric corrections from VandenBerg \& Clem (2003). We excluded FGK stars lacking a $V$ magnitude and M-dwarfs lacking an $r$ magnitude. We use these luminosities and our $T_{\text {eff }}$ values (above) to create an H-R digram of the cluster, including the planet hosts, which we show in Figure 3 alongside the model H-R diagram from Baraffe et al. (2015).

Rotation periods: following the procedures described in Gaidos et al. (2017), we attempted to compute rotation periods for 908 Praesepe candidates observed by K2. We downloaded K2SFF light curves, which we normalized and fit and subtracted off a second-order polynomial fit to each using robust methods. Rotation periods were calculated using both a Lomb-Scargle periodogram (Scargle 1981) and the autocorrelation function (ACF). The ACF value, if available, was preferred over the Lomb-Scargle value because the former is more robust to changes in light curve shape (McQuillan et al. 2013). Because the distributions of star spots often generate a second harmonic of the rotation period in the light curve, only the first and second peaks in the ACF were considered, and the higher of the two peaks was selected as the rotation period. To obtain a refined estimate of the period, a Gaussian function was fit to the ACF around the peak. Rotation periods $<35 \mathrm{~d}$ were successfully estimated for 
738 stars, including all seven host stars; other stars exhibited no significant periodic variability or an ambiguous period. We exclude another 37 stars due to questionable $r$ or $K$ magnitudes. From the remaining 701 stars, 27 have independent rotation period measurements from Agüeros et al. (2011), only one of which differs from our own measurements by more than expected errors. We show the distribution of rotation periods of the 701 stars with mass and color in Figure 4.

$v \sin i_{*}$ : projected rotational velocities were measured using our high-resolution IGRINS data. We obtained a BT-SETTL model spectrum for each target consistent with the stellar parameters derived above. The model spectra were broadened by a Gaussian to match the instrument profile, which we measured from width of the telluric lines, as described in Mann et al. (2016a). For each IGRINS order with $\mathrm{S} / \mathrm{N}>20$, we simultaneously fit for $v \sin i_{*}$ and five other nuisance parameters to handle flux calibration, wavelength calibration, and imperfectly corrected telluric lines. We assumed a microturbulent velocity of $1.5 \mathrm{~km} \mathrm{~s}^{-1}$ for all stars, and linear limbdarkening coefficients derived from PHOENIX models (see Section 4) matching the parameters of each target. After fitting, we identify large $\gg 5 \sigma$ outliers in the residuals, which we mask out and repeat the process. Most of these outlier regions appear to be missing/erroneous lines in the model spectra or poorly corrected tellurics.

For our final $v \sin i_{*}$ we adopted the robust weighted mean and standard error across all orders. Based on a comparison between our own measurements of $v \sin i_{*}$ and those in the literature of the same young stars (e.g., Mermilliod et al. 2009; Dahm et al. 2012) we add an additional systematic error of $0.6 \mathrm{~km} \mathrm{~s}^{-1}$. We attribute

\section{TRANSIT FITTING}

We fit all K2 light curves with a Monte Carlo Markov Chain (MCMC) as described in Mann et al. (2016a), which we briefly summarize here. We used the emcee Python module (Foreman-Mackey et al. 2013) to fit the model light curves produced by the batman package (Kreidberg 2015) using the Mandel \& Agol (2002) algorithm. Following Kipping (2010) we over-sampled and binned the model to match the 30 minute $K 2$ cadence. We sampled the planet-to-star radius ratio $\left(R_{P} / R_{*}\right)$, impact parameter $(b)$, orbital period $(P)$, epoch of the first transit mid-point $\left(T_{0}\right)$, bulk stellar density $\left(\rho_{*}\right)$, two parameters that describe the eccentricity and argument of periastron $(\sqrt{e} \sin \omega$ and $\sqrt{e} \cos \omega)$, and two (quadratic) limb-darkening parameters ( $q 1$ and $q 2)$.

We assumed a quadratic limb-darkening law and use the triangular sampling method of Kipping (2013) in order to uniformly sample the physically allowed region of parameter space. We applied a prior on limb-darkening this extra error to poorly understood systematics, such as micro- or macroturbulence, improperly corrected instrumental broadening, and imperfect limb-darkening corrections. Because of the limiting resolution of the spectrograph, we consider measurements below $2.6 \mathrm{~km} \mathrm{~s}^{-1}$ to be upper limits, which is the case for three targets. Based on their radii and rotation periods, all three have equatorial velocities below this limit, so only upper limits are expected.

Sky projected stellar inclination: $i_{*}$ can be compared to the planetary inclination $(i)$ measured from the transit (usually $\simeq 90^{\circ}$ ) as a probe of the planetary spin-orbit alignment. This in turn can be used to probe the formation or dynamical history of the planetary system. While this method is not as accurate as measurements from asteroseismology (e.g., Huber et al. 2013) or RossiterMcLaughlin (e.g. Narita et al. 2010), it is still sufficient to identify highly misaligned systems and can provide meaningful constraints when applied to populations of planet hosts (e.g., Walkowicz \& Basri 2013). Furthermore, $v \sin i_{*}$ and $P_{\text {rot }}$, and therefore $i_{*}$ are generally more easily measured in rapidly rotating stars like those in this study.

We calculate $i_{*}$ from $v \sin i_{*}, P_{\text {rot }}$, and $R_{*}$ using the formalism from Morton \& Winn (2014). For targets with only upper limits on $v \sin i_{*}$ we do not attempt to derive $i_{*}$. We ignored effects from differential rotation. To handle regions where $v \sin i_{*}>V_{\text {eq }}$ (which is unphysical), we converted $v \sin i_{*}$ and $V_{\text {eq }}$ to a posterior in $\cos \left(i_{*}\right)$. In all other cases, the resulting $i_{*}$ posteriors only provide lower limits on $i_{*}$ because they are all consistent with spin-orbit alignment.

A summary of all derived stellar parameters and errors is given in Table 2 .

derived from the Husser et al. (2013) atmospheric models, calculated using the LDTK toolkit (Parviainen \& Aigrain 2015), which enabled us to account for errors in stellar parameters. For this, we used the filter and CCD transmission function for Kepler from the Kepler science center $^{3}$ and stellar parameters and errors derived in Section 3. Errors on the limb-darkening coefficients were broadened to account for model uncertainties (estimated by comparing limb-darkening parameters from different model grids). Typical resulting errors on $u 1$ and $u 2$ were 0.08 and 0.04 , respectively.

For each system (excluding EPIC 211901114b, see below), we ran two MCMC fits. For the first, we fixed $e$ and $\omega$ to zero, and used a uniform prior on $\rho$, and for the second fit we allowed $\sqrt{e} \sin \omega$ and $\sqrt{e} \cos w$ to float from 0 to 1 under uniform priors and put a Gaussian

\footnotetext{
${ }^{3}$ http://keplergo.arc.nasa.gov/CalibrationResponse.shtml
} 


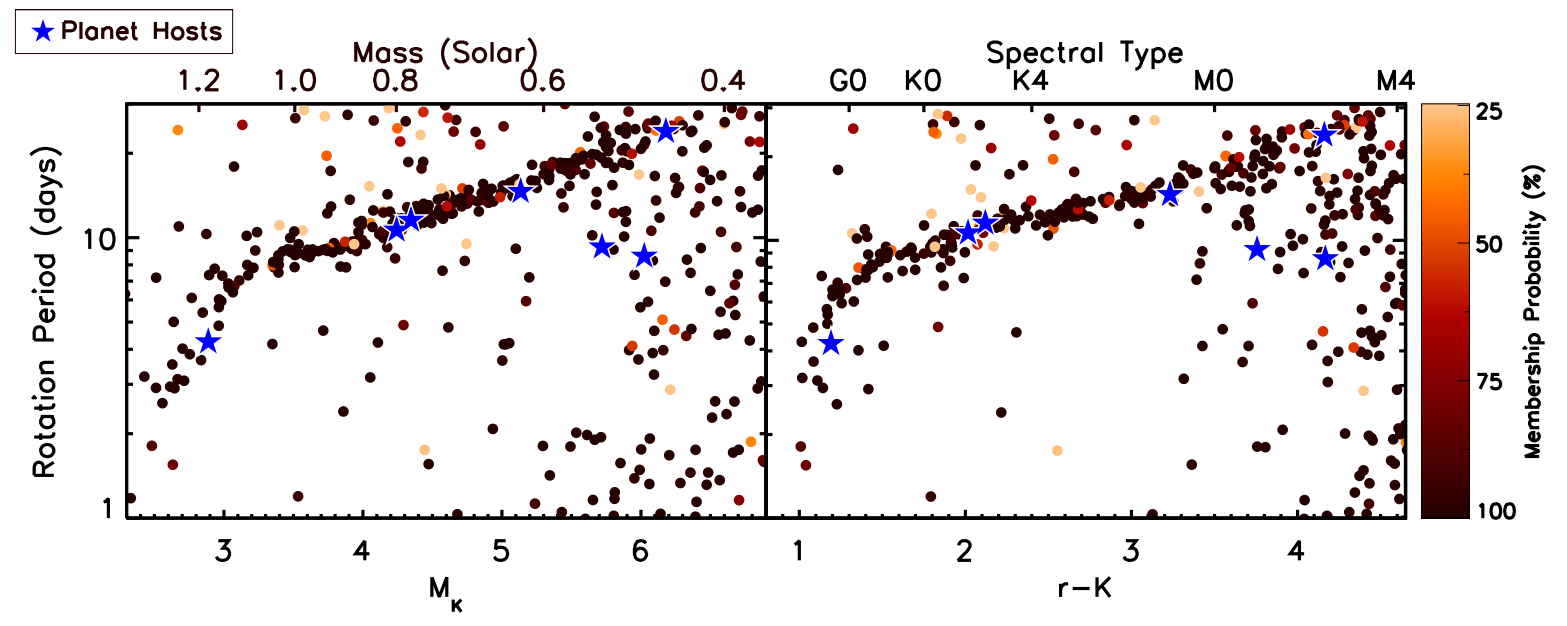

Figure 4. Rotation periods of likely Praesepe members drawn from Kraus \& Hillenbrand (2007) as a function of $M_{K}$ (left) and $r-K$ color (right). Approximate stellar masses (left) and spectral types (right) are shown on the top axes. Points are color-coded by their membership probabilities (most of which are $\simeq 100 \%$ ). The planet hosts are shown as blue stars, all of which have $\simeq 100 \%$ membership probabilities. Rotation periods are determined from an autocorrelation function, or Lomb-Scargle periodogram where autocorrelation fails. The sequence is relatively tight for $M_{K} \lesssim 5.5, r-K \lesssim 3.5$; many of the outliers in this range are likely due to binarity, non-member interlopers, differential rotation, and/or poor detection or detection of the alias of the true rotation period (e.g., Douglas et al. 2016).

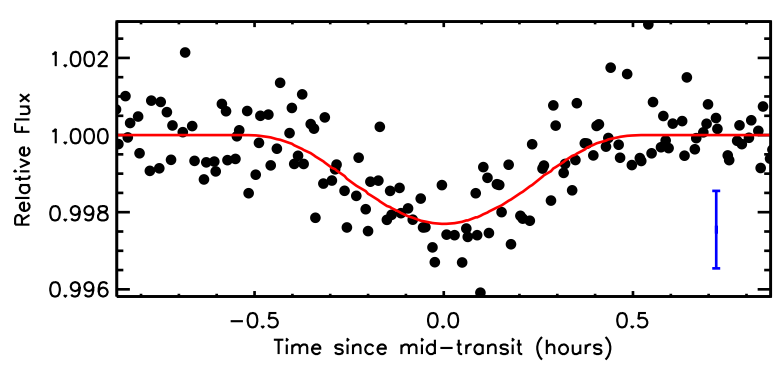

Figure 5. Phase-folded light curve of EPIC 211901114b from K2 (black points). The best-fit (highest likelihood) transit model is shown as a red line and an estimate of the photometric errors on each point is shown in the bottom right in blue.

prior on $\rho$ using our stellar parameters from Section 3. In both cases $\rho$ is forced to be $>0$, but has no upper bound.

For EPIC 211901114b the transit duration is comparable to or less than the Kepler long-cadence integration time (30 min, see Figure 5). The light curve can therefore provide only an upper limit on transit duration (or a lower limit on stellar density for $e=0$ ) without additional constraints. To mitigate this, we fit the transit with $e$ and $\omega$ fixed at zero and simultaneously apply a Gaussian prior on $\rho$. Since the planet could be truly eccentric, assuming $e=0$ may bias the resulting fit parameters. However, even with the $e=0$ constraint, the final transit-fit parameters are still highly uncertain, so it is unavoidable if we want to make any inferences about the planet. We urge caution when interpreting the transit fit for this system.
All MCMC fits were allowed to explore $|b|<1+R_{P} / R_{*}$, $P$ from 0 to 35 days, $R_{P} / R_{*}$ from 0 to 0.5 , and $T_{0}$ within $P / 2$ of the initial value, under uniform priors. For all targets, $R_{P} / R_{*}>0.5$ is conservatively ruled out by the lack of a second set of lines in our IGRINS spectrum and their locations on a color-magnitude diagram (Figure 2). All parameters were initialized to the values from our BLS search (Section 2.1), which are based on a Levenberg-Marquardt fit to the light curve (Markwardt 2009). MCMC chains were run using 150 walkers, each with 150,000 steps including a burn-in phase of 15,000 steps that was stripped from the final posteriors. Examination of the final posterior distribution suggests our selected numbers of steps, walkers, and burn-in length are more than sufficient for convergence.

We report the transit-fit parameters in Table 3. For each parameter, we report the median value with the errors as the 84.1 and 15.9 percentile values (corresponding to $1 \sigma$ for Gaussian distributions). The model light curves with the best-fit models for six of the seven systems shown in Figure 6, with EPIC 211901114 shown in Figure 5. We also show the distributions and correlations for a subset of parameters $\left(\rho, e, b\right.$, and $\left.R_{P} / R_{*}\right)$ in Figure 7 (excluding EPIC 211901114b) with the median and statistical mode for each parameter marked.

\section{FALSE-POSITIVE ANALYSIS}

We estimated the likelihood that a given candidate is a true planet using the vespa software (Morton 2012, 2015). vespa considers three astrophysical false-positive scenarios; background eclipsing binaries, bound eclipsing binaries, hierarchical eclipsing systems, and each of the 
Table 2. Stellar Parameters

\begin{tabular}{|c|c|c|c|c|c|c|c|}
\hline$\alpha$ R.A. (hh:mm:ss) & $08: 38: 24.302$ & $08: 41: 22.581$ & $08: 40: 13.451$ & $08: 41: 38.485$ & $08: 38: 32.821$ & $08: 41: 35.695$ & $08: 37: 27.058$ \\
\hline$\mu_{\alpha}\left(\operatorname{mas} \mathrm{yr}^{-1}\right)$ & $-35.7 \pm 0.6$ & $-34.3 \pm 1.8$ & $-37.1 \pm 3.0$ & $-36.4 \pm 2.5$ & $-34.7 \pm 3.9$ & $-34.0 \pm 3.0$ & $-36.0 \pm 3.0$ \\
\hline$\mu \delta\left({\left.\operatorname{mas~} \mathrm{yr}^{-1}\right)}^{-1}\right.$ & $-13.1 \pm 0.6$ & $-9.6 \pm 2.1$ & $-14.3 \pm 2.0$ & $-11.8 \pm 2.7$ & $-6.5 \pm 4.0$ & $-11.0 \pm 3.0$ & $-13.0 \pm 3.0$ \\
\hline$r$ Source & APASS & APASS & APASS & SDSS & SDSS & SDSS & SDSS \\
\hline$J$ (mag) & $9.46 \pm 0.03$ & $11.16 \pm 0.02$ & $11.28 \pm 0.02$ & $12.28 \pm 0.03$ & $12.88 \pm 0.03$ & $13.15 \pm 0.02$ & $13.31 \pm 0.02$ \\
\hline$H$ (mag) & $9.24 \pm 0.03$ & $10.68 \pm 0.02$ & $10.74 \pm 0.02$ & $11.61 \pm 0.03$ & $12.25 \pm 0.02$ & $12.54 \pm 0.02$ & $12.74 \pm 0.02$ \\
\hline$K_{S}(\mathrm{mag})$ & $9.18 \pm 0.02$ & $10.54 \pm 0.02$ & $10.64 \pm 0.02$ & $11.43 \pm 0.02$ & $12.01 \pm 0.02$ & $12.32 \pm 0.02$ & $12.47 \pm 0.02$ \\
\hline Rotation Period (days) & $4.3 \pm 0.1$ & $10.6 \pm 0.6$ & $11.5 \pm 0.7$ & $14.6 \pm 1.1$ & $9.3 \pm 0.4$ & $8.6 \pm 0.4$ & $23.9 \pm 2.4$ \\
\hline$M_{*}\left(M_{\odot}\right)$ & $1.18 \pm 0.09$ & $0.80 \pm 0.06$ & $0.77 \pm 0.06$ & $0.61 \pm 0.02$ & $0.51 \pm 0.02$ & $0.46 \pm 0.02$ & $0.43 \pm 0.02$ \\
\hline$R_{*}\left(R_{\odot}\right)$ & $1.19 \pm 0.05$ & $0.73 \pm 0.03$ & $0.71 \pm 0.03$ & $0.59 \pm 0.03$ & $0.48 \pm 0.02$ & $0.46 \pm 0.02$ & $0.44 \pm 0.02$ \\
\hline$L_{*}\left(L_{\odot}\right)$ & $1.777 \pm 0.062$ & $0.2542 \pm 0.0093$ & $0.2201 \pm 0.0082$ & $0.0703 \pm 0.0021$ & $0.0368 \pm 0.0012$ & $0.0268 \pm 0.0010$ & $0.0232 \pm 0.0009$ \\
\hline$\rho_{*}\left(\rho_{\odot}\right)$ & $0.70_{-0.09}^{+0.11}$ & $2.07_{-0.25}^{+0.29}$ & $2.14_{-0.27}^{+0.31}$ & $2.98_{-0.38}^{+0.43}$ & $4.64_{-0.60}^{+0.68}$ & $4.62_{-0.60}^{+0.69}$ & $5.16_{-0.67}^{+0.77}$ \\
\hline
\end{tabular}

Note-All $J H K_{S}$ magnitudes are from 2MASS.
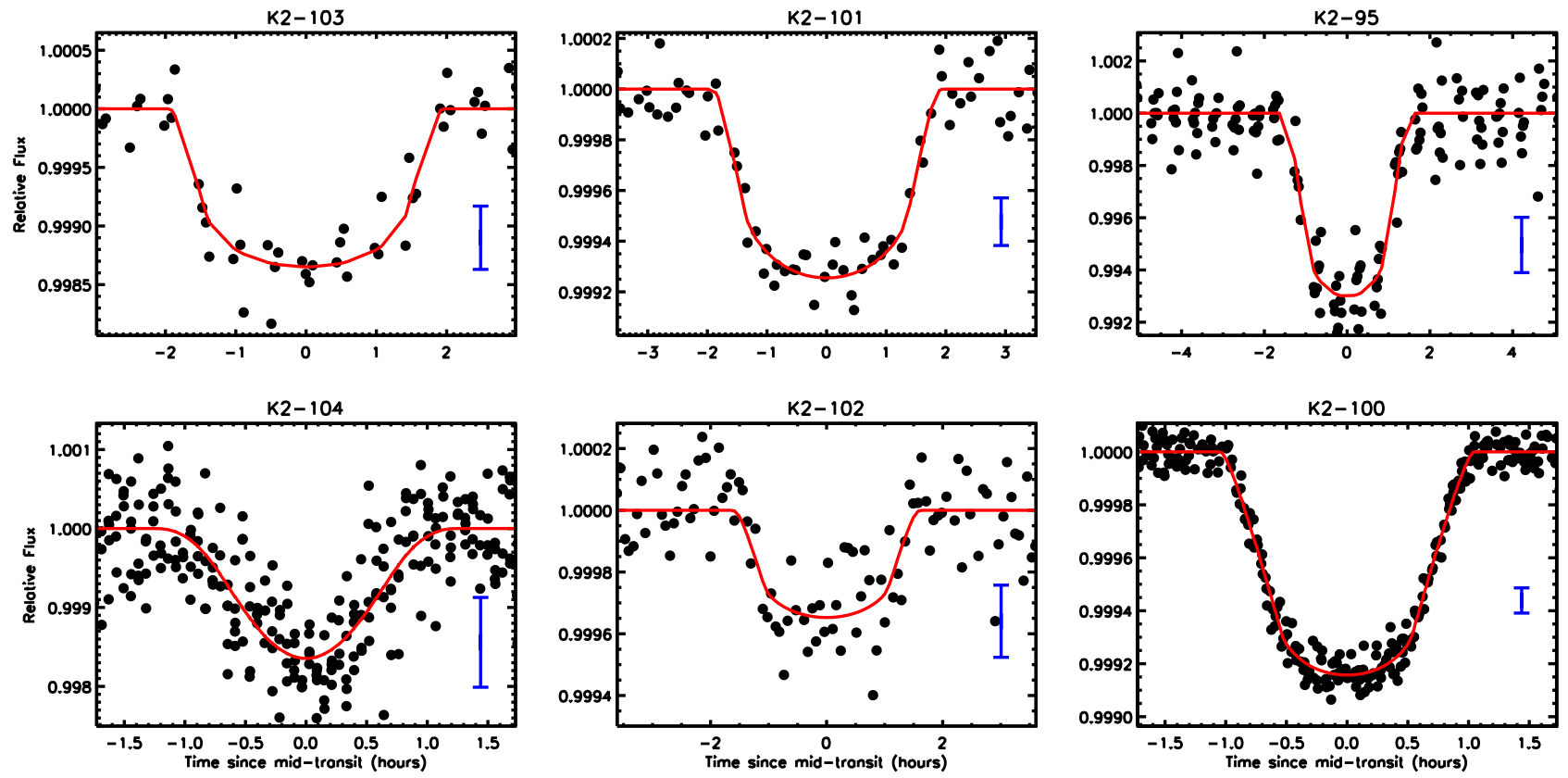

Figure 6. Phase-folded light curve of six of the transiting planets from K2 (black points). The best-fit (highest likelihood) transit models are shown as red solid lines. Estimates of the photometric errors for each star are shown as blue error bars in the bottom right corner of each panel. 

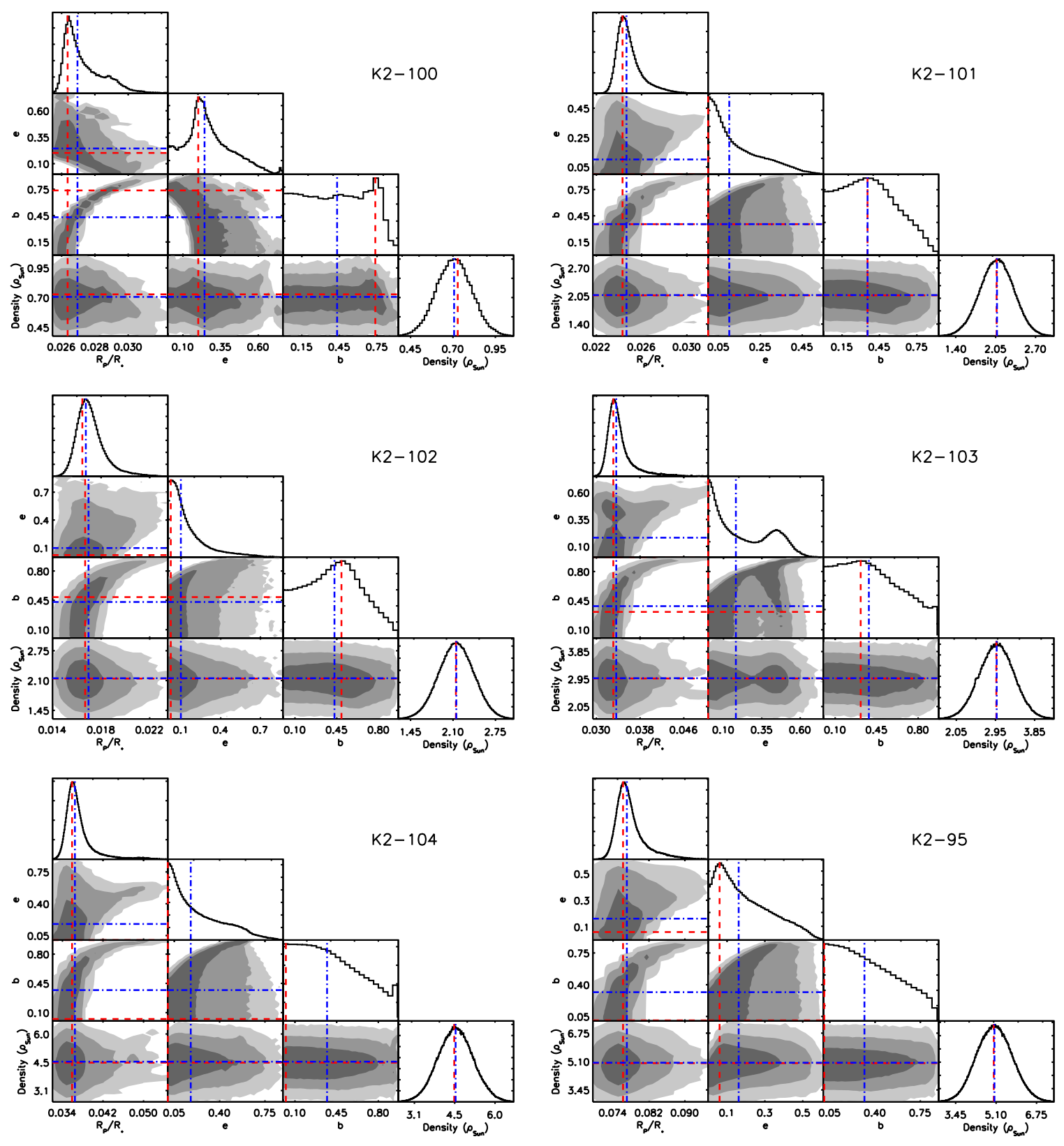

Figure 7. Distributions and correlations between $\rho, e, b$, and $R_{P} / R_{*}$ for the six systems where $e$ was allowed to float (so EPIC 211901114 is excluded). The gray regions contain $68 \%, 95 \%$, and $99.7 \%$ of the points (from darkest to lightest). MCMC steps with $b<0$ are allowed in our MCMC, but the posteriors are symmetric about $b=0$ so we instead show $|b|$. The red dashed lines mark the statistical mode and the blue dotted-dashed blue lines correspond to the median of each distribution. Plot ranges exclude a small fraction of the points $(<1 \%)$, so more clarity can be seen in the main distribution. 


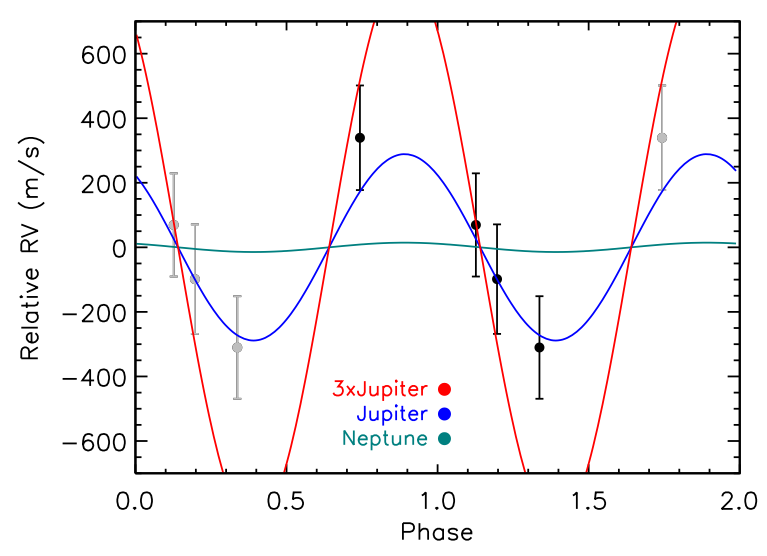

Figure 8. Radial velocities for EPIC 211901114 phased to the candidate planet's orbital period. Gray points are repeated points. The expected variation due to Neptune, Jupiter, and $3 x$ Jupiter mass planets are shown as teal, blue, and red lines, all assuming circular orbits.

three but at double the reported period. vespa then compares the likelihood of each false-positive scenario to that of a planet accounting for the shape and depth of the transit, the properties of the star, and external constraints from our AO imaging (where available). All FPP values are listed in Table 3 .

With the exception of EPIC 211901114b, all candidates are assigned false-positive probabilities (FPP) of $<1 \%$, effectively confirming their planetary nature. EPIC 211901114b was initially assigned an ambiguous FPP of $36 \%$, owing primarily to a high probability of being an eclipsing binary (34\%). This is consistent with own transit-fit posterior, which does not rule out a stellar or brown dwarf radius $\left(>11 R_{\oplus}\right)$. Our IGRINS-derived radial velocity measurements are consistent with no variation, ruling out any companion $>$ 5Jupiter masses at 5-sigma at the planet candidate's orbital period and assuming a circular orbit (Figure 8). This reduces the FPP to (2\%); the remaining FPP is due to the possibility that the system is a hierarchical eclipsing binary. The IGRINS spectra show only one set of lines and there is no evidence of an unresolved binary in the CMD position of EPIC 211901114, but these cannot rule out a companion eclipsing binary significantly fainter than the primary $(\Delta K>3$ mags). We conservatively consider EPIC 211901114b unconfirmed, pending additional AO observations or higher cadence transit observations.

vespa does not consider false-positives due to instrumental correlated ('red') noise, which may be significant for K2, nor does it consider stellar variability (spots), which is non-negligible for such young stars. We reject the first scenario for a number of reasons. First, with the exception of EPIC 211901114, all light curves show a transit-like shape, with a visible limb-darkened ingress, egress, and flattening bottom. The bottom of K2-104's light curve appears more $\mathrm{V}$-shaped due to a short duration and significant limb-darkening, but this is consistent with expectations given the period and stellar parameters. Furthermore, none of the planets have orbital periods consistent with an alias of the K2 drift or thruster fire timescale. Lastly, all planets are detected in at least two of the publicly available reduced K2 light curves: K2SC (Aigrain et al. 2016), EVEREST (Luger et al. 2016), and/or K2SFF (Vanderburg \& Johnson 2014), and all are detected in our own extracted curves. This consistency suggests that no signal is an artifact of the data reduction process.

We similarly reject the possibility that these signals are due to stellar variability. Flaring can be seen on some host light curves, including near transit, but all transits are detected even when data points near stellar flares are removed. The transit durations and shapes are inconsistent with any reasonable spot pattern. Most importantly, no planet has a period consistent with an alias of the measured rotation period, indicating that the two signals are independent of each other.

Another scenario not directly considered by our vespa analysis is a blended planet, i.e., a bound or background star with a transiting planet creating the signal. In the case of a bound companion, or background star that is a member of the cluster, the planet would still orbit a member of Praesepe, so we do not consider these false-positives. However, our derived planet parameters would be incorrect due to incorrect stellar parameters and significant uncorrected dilution from the primary star. For a non-cluster member as the transit source, analysis of Kepler planet candidates suggests cases of background transiting planets are intrinsically rare (1-4\% Fressin et al. 2013). In either case, the star with the planet (bound or background) would need to be similar in brightness to the target to reproduce the transit depth, and would likely be seen as a higher CMD position, a second set of lines in the IGRINS spectra, or a companion in the AO/NRM data if available.

\section{DISCUSSION}

\subsection{The Dynamical State of Close-in Planets at $\simeq 800$ Myr}

All systems are consistent with zero or low eccentricities $(\simeq 0.2)$ and alignment with their host star's rotation. This matches findings for the Hyades planet (K2-25b, Mann et al. 2016a; David et al. 2016b). Of the seven systems (including K2-25b, but excluding EPIC 211901114b) K2-100b has the transit-fit posterior most consistent with a non-zero $e$, but can be reconciled with a larger impact parameter (see Figure 7). Similarly, all $v \sin i_{*}$ measurements are within expectations (given uncertainties) for spin-orbit aligned systems. Even those 
with only upper limits on $v \sin i_{*}$ are expected to have equatorial velocities well below IGRINS resolution.

Unfortunately, our eccentricity measurements are all quite coarse (typical errors of 0.1-0.2), making it difficult to rule out small but non-zero eccentricities. However, the distribution of $e$ with $\omega$ in the transit-fit posteriors suggests the underlying eccentricity distribution of all systems is smaller than when considering each system individually. The value of $\rho_{*}$ derived from a transit light curve assuming zero eccentricity versus letting $e$ float will differ by a factor that depends on $\omega$ and $e$ (Seager \& Mallén-Ornelas 2003; Kipping et al. 2012):

$$
\rho_{*}^{\operatorname{circ}} \simeq\left(\frac{1+e \sin \omega}{\left(1-e^{2}\right)^{1 / 2}}\right)^{3} \rho_{*} .
$$

The factor in front of $\rho_{*}$ can be $\simeq 1$ for $e \simeq 0$ or when $\sin \omega \simeq \frac{\left(1-e^{2}\right)^{1 / 2}-1}{e}$. Thus fits to the light curve of a transiting planet with $e_{\text {true }}=0$ can often yield answers with $e_{\text {measured }} \gg 0$ at specific values of $\omega$, especially in the presence of red and white noise (Pont et al. 2006; Gazak et al. 2012). This effect can be seen in our own fit posteriors, of which we show an example in Figure 9. A similar pattern is seen in all fits where $e$ is not fixed to zero: values of $e \gg 0$ tend to be clustered around specific values of $\omega$ that keep the transit observables unchanged. For near-circular orbits $\omega$ should be $\simeq$ random, so the true $e$ distribution is likely smaller than the combined posteriors imply.

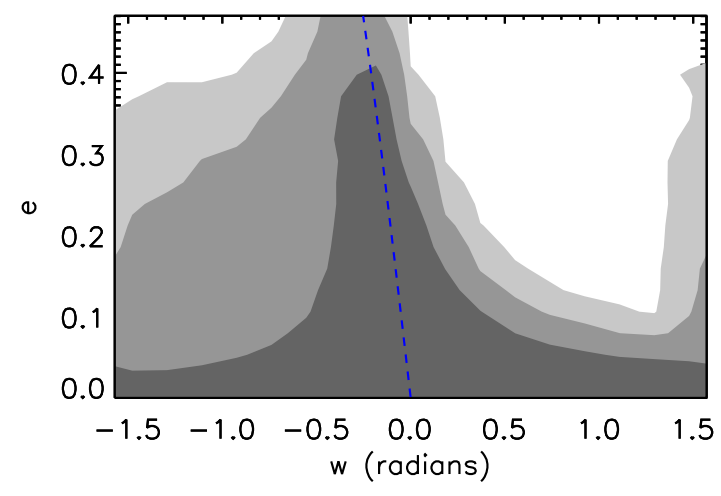

Figure 9. Correlation between $\omega$ and $e$ for K2-101b following the shading scheme of Figure 7 . The blue dashed line marks where $\rho_{*}^{\text {circ }} \simeq \rho_{*}$ and $e \neq 0$, a region where large values of $e$ are allowed with minimal impact on the transit shape.

A solution to this is to generate simulated $e$ and $\omega$ distributions and compare them to the distribution of $\rho_{\text {transit }} / \rho_{*}$ derived from our fits with $e=0$ and our stellar parameters from Section 3, similar to the procedure in Van Eylen \& Albrecht (2015). Assuming a Rayleigh distribution for $e$ and a uniform distribution in $\omega$, we can set an upper limit on the Rayleigh scale parameter $\left(\sigma_{e}\right)$ of 0.26 at $95 \%$. A more sophisticated treatment would also account for the selection bias in favor of higher eccentricity systems (Kipping 2014), but this effect is smaller than current errors. Additional systems and higher cadence ground-based transit photometry would provide significantly improved constraints.

The spin-orbit alignment measurements, similar to measurements of $e$, provide only rough constraints. For the four systems where we detect $v \sin i_{*}$ broadening the measurements are consistent with alignment. Although $v \sin i_{*}$ is too small to detect in the other three systems, non-detection is expected based on their rotation periods and radii. Higher resolution observations would help with the missing systems, and measurements of the RossiterMcLaughlin effect could provide significantly stronger constraints on spin-orbit alignment (e.g. Narita et al. 2010). In cases where the Rossiter-McLaughlin effect is too small to detect (due to small $v \sin i_{*}$ and transit depth) it may be possible to measure spin-orbit alignment by observing spot-crossing events (e.g., Nutzman et al. 2011).

Studies of transiting planets around older ( $\gtrsim 1 \mathrm{Gyr})$ stars from Kepler suggests Earth- to Neptune-size planets have small $(\lesssim 0.1)$ eccentricities (Hadden \& Lithwick 2014; Van Eylen \& Albrecht 2015), and their orbits are generally aligned with their host star (e.g., Walkowicz \& Basri 2013). However, after dissipation of the protoplanetary gas disk, planet-planet scattering can drive super-Earth to Neptune size planets to large eccentricities $(\gg 0.4)$ and spin-orbit misalignment on 100 Myr timescales (e.g., Chatterjee et al. 2008; Kennedy \& Kenyon 2008). While coarse, our findings suggest that such scattering or other highly disruptive events in young planetary systems are not the norm, and at 800 Myr systems may be as dynamically settled as their old counterparts.

Another tool used to probe the dynamical state of planetary systems is the number of multiplanet systems, which provides constraints on the level of mutual inclination of planets (e.g., Ballard \& Johnson 2016). We detect no multiplanet system, yet approximately $22 \%$ of Kepler systems are known to harbor multiple planets (Batalha 2014). This suggests that, of the 8 systems in Hyades and Praesepe we should have found $1.8 \mathrm{mul}-$ tiplanet systems, with a Poisson probability of $17 \%$ of detecting none. If we cut the Kepler sample on $\mathrm{S} / \mathrm{N}$, period, and host star properties to simulate a K2 like survey of Praesepe $(P<35$ days, fewer detected transits, only dwarf stars, etc.) then the fraction of multiples drops to $16 \%$, and the Poisson probability increases to $28 \%$ of finding no multiplanet systems. So there is not a statistically significant deficit of multiples and we can draw no useful conclusions about the mutual inclination distribution of young planets at this time. 


\subsection{Why Are There So Few Planets in the Pleiades?}

There is a notable difference in the number of planets detected in the $\simeq 800$ Myr old Praesepe and Hyades clusters (8 planets) versus in the $\simeq 125$ Myr old Pleiades cluster (0 planets, Gaidos et al. 2017). This is despite the fact that the target samples are similar in number $(\simeq 1000$ targets in Praesepe + Hyades and $\simeq 1000$ in Pleiades), spectral type distribution, and metallicity. Furthermore, Pleiades is closer than Praesepe (136 pc versus 181 pc), and hence similar mass stars are statistically brighter. It is possible that we are seeing signatures of planetary migration on 0.1-1 Gyr timescales, but the differences are not yet statistically significant.

The difference in number of planets detected between the clusters may instead be due to faster and higher amplitude rotation for younger Pleiades stars, which can be difficult to remove and complicate the detection of short-period planets. A planet injection test assuming a Kepler-like population done by Gaidos et al. (2017) indicates this is at least partially to blame for the lack of detections in Pleiades. Typical rotation periods in Pleiades are $1 \mathrm{~d}<P_{\text {rot }}<10 \mathrm{~d}$ for the range of spectral types probed by K2 (Covey et al. 2016). We detect three planets around stars with $P_{\text {rot }}<10$ days in Praesepe, but none around stars with $P_{\text {rot }}<4$ days (Figure 4). However, many fast rotating stars ( $P_{\text {rot }}<4$ days) in Praesepe are likely to be tidally locked binaries (Douglas et al. 2016), around which close-in planets are significantly less common (Wang et al. 2014; Kraus et al. 2016). So the lack of detections around the fastest rotators is not conclusive.

A more detailed injection/recovery test across all nearby young clusters/star-forming regions (Upper Scorpius, Hyades, Pleiades, and Praesepe) to better constrain our completeness as a function of rotation period (and other stellar properties) would be useful, particularly when combined with the additional $K \mathscr{2}$ observations of Hyades planned for Campaign 13. TESS is also expected to survey nearby young stars and clusters (Stassun et al. 2014) and could shed significant light on this difference.

\subsection{Are Young Planets Larger than their Older Counterparts?}

Mann et al. (2016a) found that the $\simeq 800 \mathrm{Myr}$ old Hyades planet K2-25b has an unusually large radius when compared to other transiting planets from Kepler given its host star mass and incident flux. Higher-mass stars have larger disk masses (Andrews et al. 2013; Pascucci et al. 2016) and hence are more likely to harbor large planets (e.g. Johnson et al. 2010; Mulders et al. 2015), and planets that receive more flux from their host star may lose their atmospheres faster (we use bolometric flux as a proxy for high-energy flux). Thus such large planetary radii suggest that, at this age, close-in planets around M-dwarfs may still be losing atmosphere due to interaction with their host star.

We show a comparison of planet size as a function of host star mass and incident flux for M-dwarf planets drawn from transit surveys in Figure 10. Stellar and planetary parameters for Kepler systems are taken from Gaidos et al. (2016), MEarth systems from AngladaEscudé et al. (2013) and Berta-Thompson et al. (2015), the Hyades system from Mann et al. (2016a), and the Upper Scorpius system from Mann et al. (2016b). Planets identified through RV surveys are not included becuase they have different selection criteria and observational biases, though GJ 436b and GJ 3470b are notable because they also have relatively large radii $\left(\simeq 4 R_{\oplus}\right)$ despite orbiting old (> $1 \mathrm{Gyr}$ ) stars (von Braun et al. 2012; Biddle et al. 2014). GJ 436b, interestingly, also shows evidence of an evaporating atmosphere (Ehrenreich et al. 2015).

Excluding EPIC 211901114b, whose parameters are poorly constrained, only one target of six, K2-95b, is significantly larger than the $R_{P}-M_{*}$ sequence from Kepler, with a radius of $3.7 \pm 0.2 \mathrm{R}_{\oplus}$ and host star mass of $M_{*}=0.430 \pm 0.02 M_{\odot}$, as noted by Obermeier et al. (2016). Although K2-95b looks like less of an outlier given its level of incident flux. The other planets around M-dwarfs are less remarkable in terms of their size. The more typical size for K2-104b may be due to a much higher level of flux from the host star stripping the atmosphere away faster than $800 \mathrm{Myr}$. Close-in $\simeq 1 R_{\oplus}$ planets, which are common in the Kepler M-dwarf sample (Dressing \& Charbonneau 2013, 2015), are likely missing from our sample due to detection limits; Praesepe targets are statistically more distant/faint than those observed by Kepler (Gaidos \& Mann 2013), and the shorter observing window yields fewer transits and $\mathrm{S} / \mathrm{N}$. It is still suggestive that 2-3 (depending on the status of EPIC 211901114b) of the 5 known planets orbiting $<800$ Myr old M-dwarfs are large compared to 1-2 planets out of 90 orbiting old M-dwarfs in the Kepler-prime field. Mass determinations of these planets would be useful to determine if they have lower densities than their older counterparts, which would favor a scenario where close-in planets continue to lose atmosphere past 800 Myr.

The authors thank Tim Morton for his help with the vespa software. We also thank Marshall Johnson for his help with the transit fits.

AWM was supported through Hubble Fellowship grant 51364 awarded by the Space Telescope Science Institute, which is operated by the Association of Universities for Research in Astronomy, Inc., for NASA, under contract 


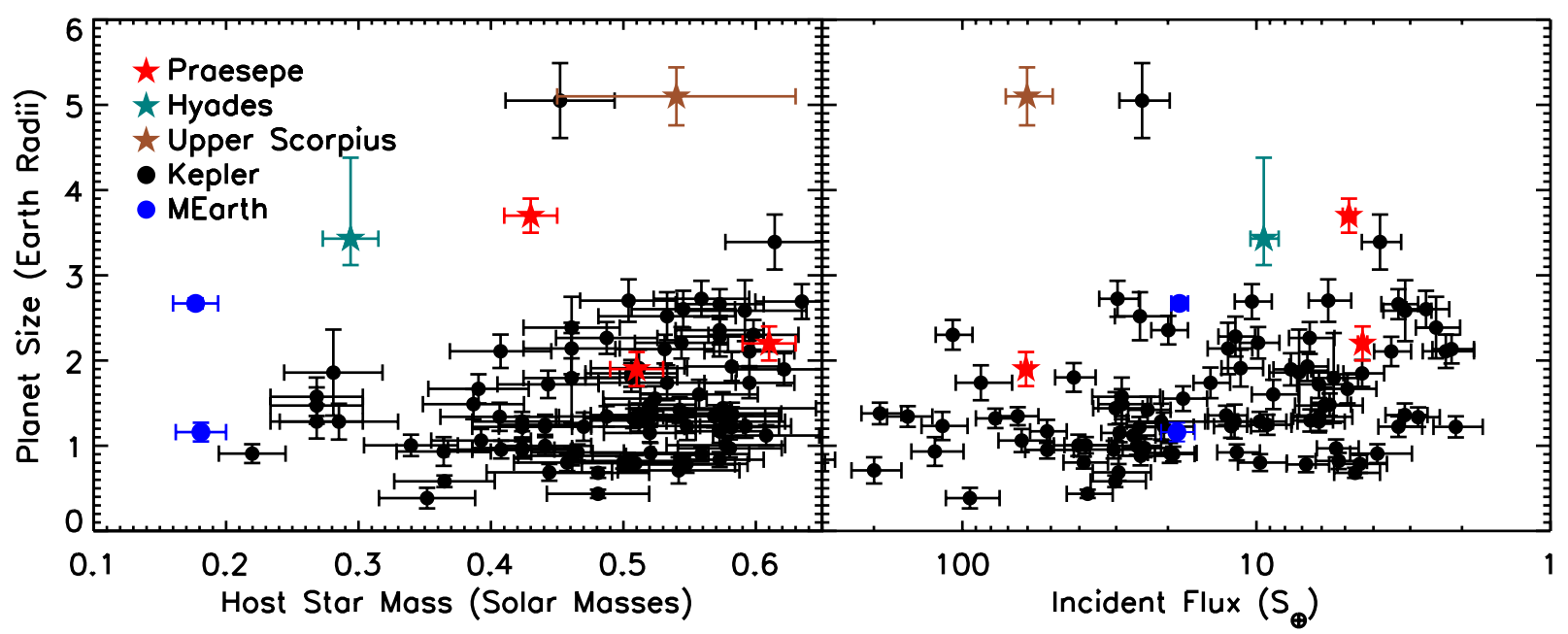

Figure 10. Planet size versus stellar mass (left) and incident flux on the planet (right) for transiting planets orbiting $M_{*}<0.65 M_{\odot}$ stars and with orbital periods $<30$ days taken from the transit surveys MEarth, K2 and Kepler. Young systems are shown as stars while older ones are circles. K2-33b (Upper Scorpius) appears inflated, but is much younger ( 11 Myr versus 800 Myr) than systems considered here. Both K2-25b (Hyades) and K2-95b are atypically large given their host star mass. The other two Praesepe planets do not appear inflated, though for one (K2-104) this may be due to a higher incident flux. EPIC 211901114b is excluded, despite its large assigned radius $\left(9.6_{-4.8}^{+5.3} R_{\oplus}\right)$ because of its uncertain status and parameters.

NAS 5-26555. This research was supported by NASA grant NNX11AC33G to EG. A.V. is supported by the NSF Graduate Research Fellowship, Grant No. DGE 1144152 .

This work used the Immersion Grating Infrared Spectrograph (IGRINS) that was developed under a collaboration between the University of Texas at Austin and the Korea Astronomy and Space Science Institute (KASI) with the financial support of the US National Science Foundation under grant ASTR1229522, of the University of Texas at Austin, and of the Korean GMT Project of KASI. The IGRINS pipeline package PLP was developed by Dr. Jae-Joon Lee at Korea Astronomy and Space Science Institute and Professor Soojong Pak's team at Kyung Hee University. SNIFS on the UH 2.2-m telescope is part of the Nearby Supernova Factory project, a scientific collaboration among the Centre de Recherche Astronomique de Lyon, Institut de Physique Nucléaire de Lyon, Laboratoire de Physique Nucléaire et des Hautes Energies, Lawrence Berkeley National Laboratory, Yale University, University of Bonn, Max Planck Institute for Astrophysics, Tsinghua Center for Astrophysics, and the Centre de Physique des Particules de Marseille. Some of the data presented in this paper were obtained from the Mikulski Archive for Space Telescopes (MAST). STScI is operated by the Association of Universities for Research in Astronomy, Inc., under NASA contract NAS5-26555. Support for MAST for non-HST data is provided by the NASA Office of Space Science via grant NNX09AF08G and by other grants and contracts. This research was made possible through the use of the AAVSO Photometric All-Sky Survey (APASS), funded by the Robert Martin Ayers Sciences Fund. The authors acknowledge the Texas Advanced Computing Center (TACC) at The University of Texas at Austin for providing HPC resources that have contributed to the research results reported within this paper ${ }^{4}$. These results made use of the Discovery Channel Telescope at Lowell Observatory. Lowell is a private, non-profit institution dedicated to astrophysical research and public appreciation of astronomy and operates the DCT in partnership with Boston University, the University of Maryland, the University of Toledo, Northern Arizona University and Yale University.

The authors wish to recognize and acknowledge the very significant cultural role and reverence that the summit of Maunakea has always had within the indigenous Hawaiian community. We are most fortunate to have the opportunity to conduct observations from this mountain.

Facilities: UH:2.2m (SNIFS), Keck:II (NIRC2), Smith (IGRINS), Kepler

\section{REFERENCES}

Adams, F. C., \& Laughlin, G. 2006, The Astrophysical Journal, 649,1004

Agüeros, M. A., Covey, K. R., Lemonias, J. J., et al. 2011, ApJ, 740,110 
Ahn, C. P., Alexandroff, R., Allende Prieto, C., et al. 2012, ApJS, 203, 21

Aigrain, S., Hodgkin, S., Irwin, J., et al. 2007, MNRAS, 375, 29

Aigrain, S., Parviainen, H., \& Pope, B. J. S. 2016, MNRAS, 459, 2408

Aldering, G., Adam, G., Antilogus, P., et al. 2002, in Society of Photo-Optical Instrumentation Engineers (SPIE) Conference Series, Vol. 4836, Survey and Other Telescope Technologies and Discoveries, ed. J. A. Tyson \& S. Wolff, 61-72

Allard, F., Homeier, D., \& Freytag, B. 2012, Royal Society of London Philosophical Transactions Series A, 370, 2765

Andrews, S. M., Rosenfeld, K. A., Kraus, A. L., \& Wilner, D. J. 2013, ApJ, 771, 129

Anglada-Escudé, G., Rojas-Ayala, B., Boss, A. P., Weinberger, A. J., \& Lloyd, J. P. 2013, A\&A, 551, A48

Ballard, S., \& Johnson, J. A. 2016, ApJ, 816, 66

Baraffe, I., Homeier, D., Allard, F., \& Chabrier, G. 2015, A\&A, 577, A42

Barros, S. C. C., Demangeon, O., \& Deleuil, M. 2016, A\&A, 594, A100

Batalha, N. M. 2014, Proceedings of the National Academy of Science, 111, 12647

Batalha, N. M., Borucki, W. J., Koch, D. G., et al. 2010, ApJL, 713, L109

Becker, J. C., Vanderburg, A., Adams, F. C., Rappaport, S. A., \& Schwengeler, H. M. 2015, ApJL, 812, L18

Berta-Thompson, Z. K., Irwin, J., Charbonneau, D., et al. 2015, Nature, 527, 204

Biddle, L. I., Pearson, K. A., Crossfield, I. J. M., et al. 2014, MNRAS, 443, 1810

Boesgaard, A. M., Roper, B. W., \& Lum, M. G. 2013, ApJ, 775, 58

Borucki, W. J., Koch, D., Basri, G., et al. 2010, Science, 327, 977

Boyajian, T. S., McAlister, H. A., van Belle, G., et al. 2012a, ApJ, 746, 101

Boyajian, T. S., von Braun, K., van Belle, G., et al. 2012b, ApJ, 757, 112

Brandt, T. D., \& Huang, C. X. 2015, ApJ, 807, 24

Buchhave, L. A., Latham, D. W., Johansen, A., et al. 2012, Nature, 486, 375

Cardelli, J. A., Clayton, G. C., \& Mathis, J. S. 1989, ApJ, 345, 245

Cargile, P. A., James, D. J., Pepper, J., et al. 2014, ApJ, 782, 29

Chatterjee, S., Ford, E. B., Matsumura, S., \& Rasio, F. A. 2008, ApJ, 686, 580

Cochran, W. D., Hatzes, A. P., \& Paulson, D. B. 2002, AJ, 124, 565

Cohen, M., Wheaton, W. A., \& Megeath, S. T. 2003, AJ, 126, 1090

Covey, K. R., Agüeros, M. A., Law, N. M., et al. 2016, ApJ, 822, 81

Crockett, C. J., Mahmud, N. I., Prato, L., et al. 2012, ApJ, 761, 164

Crossfield, I. J. M., Ciardi, D. R., Petigura, E. A., et al. 2016, ApJS, 226, 7

Cushing, M. C., Vacca, W. D., \& Rayner, J. T. 2004, PASP, 116, 362

Dahm, S. E. 2015, ApJ, 813, 108

Dahm, S. E., Slesnick, C. L., \& White, R. J. 2012, ApJ, 745, 56

David, T. J., Hillenbrand, L. A., Petigura, E. A., et al. 2016a, Nature, 534, 658

David, T. J., Conroy, K. E., Hillenbrand, L. A., et al. 2016b, AJ, 151,112

Delfosse, X., Forveille, T., Ségransan, D., et al. 2000, A\&A, 364, 217

Dotter, A., Chaboyer, B., Jevremović, D., et al. 2008, ApJS, 178, 89
Douglas, S. T., Agüeros, M. A., Covey, K. R., et al. 2016, ApJ, 822,47

-. 2014, ApJ, 795, 161

Dressing, C. D., \& Charbonneau, D. 2013, ApJ, 767, 95

—. 2015, ApJ, 807, 45

Ehrenreich, D., Bourrier, V., Wheatley, P. J., et al. 2015, Nature, 522,459

Feiden, G. A., \& Chaboyer, B. 2012, ApJ, 757, 42

Foreman-Mackey, D., Hogg, D. W., Lang, D., \& Goodman, J. 2013, PASP, 125, 306

Fressin, F., Torres, G., Charbonneau, D., et al. 2013, ApJ, 766, 81

Gaidos, E., Fischer, D. A., Mann, A. W., \& Howard, A. W. 2013 , ApJ, 771, 18

Gaidos, E., \& Mann, A. W. 2013, ApJ, 762, 41

Gaidos, E., Mann, A. W., Kraus, A. L., \& Ireland, M. 2016, MNRAS, 457, 2877

Gaidos, E., Mann, A. W., Lépine, S., et al. 2014, MNRAS, 443, 2561

Gaidos, E., Mann, A. W., Rizzuto, A., et al. 2017, MNRAS, 464, 850

Gáspár, A., Rieke, G. H., Su, K. Y. L., et al. 2009, ApJ, 697, 1578

Gazak, J. Z., Johnson, J. A., Tonry, J., et al. 2012, Advances in Astronomy, 2012, arXiv:1102.1036

Grunblatt, S. K., Huber, D., Gaidos, E. J., et al. 2016, AJ, 152, 185

Hadden, S., \& Lithwick, Y. 2014, ApJ, 787, 80

Hartkopf, W. I., McAlister, H. A., \& Mason, B. D. 2001, AJ, 122 , 3480

Henden, A. A., Levine, S. E., Terrell, D., Smith, T. C., \& Welch, D. 2012, Journal of the American Association of Variable Star Observers (JAAVSO), 40, 430

Henry, T. J., \& McCarthy, Jr., D. W. 1993, AJ, 106, 773

Høg, E., Fabricius, C., Makarov, V. V., et al. 2000, A\&A, 355, L27

Howell, S. B., Sobeck, C., Haas, M., et al. 2014, PASP, 126, 398

Huber, D., Carter, J. A., Barbieri, M., et al. 2013, Science, 342, 331

Husser, T.-O., Wende-von Berg, S., Dreizler, S., et al. 2013, A\&A, 553, A6

Jenkins, J. M., Caldwell, D. A., Chandrasekaran, H., et al. 2010, ApJL, 713, L120

Johnson, J. A., Aller, K. M., Howard, A. W., \& Crepp, J. R. 2010, PASP, 122, 905

Kennedy, G. M., \& Kenyon, S. J. 2008, ApJ, 682, 1264

Kipping, D. M. 2010, MNRAS, 408, 1758

-. 2013, MNRAS, 435, 2152

—. 2014, MNRAS, 444, 2263

Kipping, D. M., Dunn, W. R., Jasinski, J. M., \& Manthri, V. P. 2012, MNRAS, 421, 1166

Kovács, G., Zucker, S., \& Mazeh, T. 2002, A\&A, 391, 369

Kraus, A. L., \& Hillenbrand, L. A. 2007, AJ, 134, 2340

Kraus, A. L., Ireland, M. J., Huber, D., Mann, A. W., \& Dupuy, T. J. 2016, AJ, 152, 8

Kraus, A. L., Ireland, M. J., Martinache, F., \& Lloyd, J. P. 2008 , ApJ, 679, 762

Kreidberg, L. 2015, PASP, 127, 1161

Lantz, B., Aldering, G., Antilogus, P., et al. 2004, in Society of Photo-Optical Instrumentation Engineers (SPIE) Conference Series, Vol. 5249, Optical Design and Engineering, ed. L. Mazuray, P. J. Rogers, \& R. Wartmann, 146-155

Lee, J.-J. 2015, plp: Version 2.0, doi:10.5281/zenodo.18579

Libralato, M., Nardiello, D., Bedin, L. R., et al. 2016, MNRAS, arXiv: 1608.00459

Liu, F., Yong, D., Asplund, M., Ramírez, I., \& Meléndez, J. 2016, MNRAS, 457, 3934

Lovis, C., \& Mayor, M. 2007, A\&A, 472, 657

Luger, R., Agol, E., Kruse, E., et al. 2016, AJ, 152, 100 
Mace, G., Kim, H., Jaffe, D. T., et al. 2016, 300 Nights of Science with IGRINS at McDonald Observatory, doi:10.1117/12.2232780

Malavolta, L., Nascimbeni, V., Piotto, G., et al. 2016, A\&A, 588, A118

Malo, L., Doyon, R., Lafrenière, D., et al. 2013, ApJ, 762, 88

Mandel, K., \& Agol, E. 2002, ApJL, 580, L171

Mann, A. W., Feiden, G. A., Gaidos, E., Boyajian, T., \& von Braun, K. 2015, ApJ, 804, 64

Mann, A. W., Gaidos, E., \& Ansdell, M. 2013a, ApJ, 779, 188

Mann, A. W., Gaidos, E., Kraus, A., \& Hilton, E. J. 2013b, ApJ, 770,43

Mann, A. W., \& von Braun, K. 2015, PASP, 127, 102

Mann, A. W., Gaidos, E., Mace, G. N., et al. 2016a, ApJ, 818, 46

Mann, A. W., Newton, E. R., Rizzuto, A. C., et al. 2016b, AJ, 152,61

Markwardt, C. B. 2009, in Astronomical Society of the Pacific Conference Series, Vol. 411, Astronomical Data Analysis Software and Systems XVIII, ed. D. A. Bohlender, D. Durand, \& P. Dowler, 251

McQuillan, A., Aigrain, S., \& Mazeh, T. 2013, MNRAS, 432, 1203

Meibom, S., Torres, G., Fressin, F., et al. 2013, Nature, 499, 55

Mermilliod, J.-C., Mayor, M., \& Udry, S. 2009, A\&A, 498, 949

Mochejska, B. J., Stanek, K. Z., Sasselov, D. D., \& Szentgyorgyi, A. H. 2002, AJ, 123, 3460

Morton, T. D. 2012, ApJ, 761, 6

-. 2015, VESPA: False positive probabilities calculator, Astrophysics Source Code Library, ascl:1503.011

Morton, T. D., \& Winn, J. N. 2014, ApJ, 796, 47

Mulders, G. D., Pascucci, I., \& Apai, D. 2015, ApJ, 798, 112

Mullally, F., Coughlin, J. L., Thompson, S. E., et al. 2015, ApJS, 217,31

Narita, N., Hirano, T., Sanchis-Ojeda, R., et al. 2010, PASJ, 62, L61

Netopil, M., Paunzen, E., Heiter, U., \& Soubiran, C. 2016, A\&A, 585, A150

Neves, V., Bonfils, X., Santos, N. C., et al. 2013, A\&A, 551, A36

Nutzman, P. A., Fabrycky, D. C., \& Fortney, J. J. 2011, ApJL, 740, L10

Obermeier, C., Henning, T., Schlieder, J. E., et al. 2016, AJ, 152, 223

Park, C., Jaffe, D. T., Yuk, I.-S., et al. 2014, in Society of Photo-Optical Instrumentation Engineers (SPIE) Conference Series, Vol. 9147, Society of Photo-Optical Instrumentation Engineers (SPIE) Conference Series, 1

Parviainen, H., \& Aigrain, S. 2015, MNRAS, 453, 3821

Pascucci, I., Testi, L., Herczeg, G. J., et al. 2016, ApJ, 831, 125

Paulson, D. B., Cochran, W. D., \& Hatzes, A. P. 2004, AJ, 127, 3579

Perryman, M. A. C., Brown, A. G. A., Lebreton, Y., et al. 1998, A\&A, 331, 81

Petigura, E. A., Marcy, G. W., \& Howard, A. W. 2013, ApJ, 770, 69

Pinsonneault, M. H., An, D., Molenda-Żakowicz, J., et al. 2012, ApJS, 199, 30

Pont, F., Zucker, S., \& Queloz, D. 2006, MNRAS, 373, 231

Pope, B. J. S., Parviainen, H., \& Aigrain, S. 2016, MNRAS, 461, 3399

Quinn, S. N., White, R. J., Latham, D. W., et al. 2012, ApJL, 756, L33

-. 2014, ApJ, 787, 27

Ramírez, I., \& Meléndez, J. 2005, ApJ, 626, 465

Raymond, S. N., Barnes, R., Veras, D., et al. 2009, ApJL, 696, L98

Reiners, A., Bean, J. L., Huber, K. F., et al. 2010, ApJ, 710, 432

Rizzuto, A. C., Ireland, M. J., \& Robertson, J. G. 2011, MNRAS, 416, 3108

Salaris, M., Weiss, A., \& Percival, S. M. 2004, A\&A, 414, 163

Scargle, J. D. 1981, ApJS, 45, 1
Schneider, F. R. N., Izzard, R. G., de Mink, S. E., et al. 2014 ApJ, 780, 117

Seager, S., \& Mallén-Ornelas, G. 2003, ApJ, 585, 1038

Silva Aguirre, V., Davies, G. R., Basu, S., et al. 2015, MNRAS, 452,2127

Skrutskie, M. F., Cutri, R. M., Stiening, R., et al. 2006, AJ, 131, 1163

Stassun, K. G., Pepper, J. A., Oelkers, R., et al. 2014, ArXiv e-prints, arXiv:1410.6379

Taylor, B. J. 2006, AJ, 132, 2453

Vacca, W. D., Cushing, M. C., \& Rayner, J. T. 2003, PASP, 115, 389

Van Cleve, J. E., Howell, S. B., Smith, J. C., et al. 2016, PASP, 128, 075002

Van Eylen, V., \& Albrecht, S. 2015, ApJ, 808, 126

van Leeuwen, F. 2009, A\&A, 497, 209

van Saders, J. L., \& Gaudi, B. S. 2011, ApJ, 729, 63

VandenBerg, D. A., \& Clem, J. L. 2003, AJ, 126, 778

Vanderburg, A., \& Johnson, J. A. 2014, PASP, 126, 948

Vanderburg, A., Latham, D. W., Buchhave, L. A., et al. 2016, ApJS, 222, 14

von Braun, K., Boyajian, T. S., Kane, S. R., et al. 2012, ApJ, 753, 171

Walkowicz, L. M., \& Basri, G. S. 2013, MNRAS, 436, 1883

Wang, J., Fischer, D. A., Xie, J.-W., \& Ciardi, D. R. 2014, ApJ, 791,111

Wizinowich, P., Acton, D. S., Shelton, C., et al. 2000, PASP, 112 315

Wright, E. L., Eisenhardt, P. R. M., Mainzer, A. K., et al. 2010, AJ, 140, 1868

Yang, X. L., Chen, Y. Q., \& Zhao, G. 2015, AJ, 150, 158

Yelda, S., Lu, J. R., Ghez, A. M., et al. 2010, ApJ, 725, 331

Yuk, I.-S., Jaffe, D. T., Barnes, S., et al. 2010, in Society of Photo-Optical Instrumentation Engineers (SPIE) Conference Series, Vol. 7735, Society of Photo-Optical Instrumentation Engineers (SPIE) Conference Series

Zacharias, N., Finch, C. T., Girard, T. M., et al. 2013, AJ, 145, 44 
Table 3. Planet/Transit-fit Parameters

\begin{tabular}{|c|c|c|c|c|c|c|c|}
\hline Parameter & $\mathrm{K} 2-100 \mathrm{~b}$ & $\mathrm{~K} 2-101 \mathrm{~b}$ & $\mathrm{~K} 2-102 \mathrm{~b}$ & $\mathrm{~K} 2-103 \mathrm{~b}$ & $\mathrm{~K} 2-104 \mathrm{~b}$ & EPIC $211901114^{\mathrm{a}}$ & $\mathrm{K} 2-95 \mathrm{~b}$ \\
\hline \multicolumn{8}{|c|}{ Uniform stellar density Prior ${ }^{\mathrm{a}} ; e, \omega$ fixed at 0} \\
\hline Period (days) & $1.673915_{-0.000011}^{+0.000011}$ & $14.677303_{-0.000809}^{+0.000824}$ & $9.915651_{-0.001175}^{+0.001194}$ & $21.169687_{-0.001655}^{+0.001636}$ & $1.974189_{-0.000109}^{+0.000110}$ & $1.648932_{-0.000069}^{+0.000071}$ & $10.135097_{-0.000489}^{+0.000498}$ \\
\hline$R_{P} / R_{*}$ & $0.0267_{-0.0005}^{+0.0011}$ & $0.0247_{-0.0007}^{+0.0012}$ & $0.0169_{-0.0008}^{+0.0010}$ & $0.0335_{-0.0011}^{+0.0015}$ & $0.0365_{-0.0015}^{+0.0029}$ & $0.1912_{-0.0948}^{+0.1053}$ & $0.0771_{-0.0021}^{+0.0031}$ \\
\hline$T_{0}{ }^{\mathrm{b}}(\mathrm{BJD}-2400000)$ & $57144.06700_{-0.00027}^{+0.00027}$ & $57152.68125_{-0.00229}^{+0.00224}$ & $57139.65488_{-0.00539}^{+0.00538}$ & $57123.23787_{-0.00438}^{+0.00426}$ & $57140.38097_{-0.00257}^{+0.00252}$ & $57140.83259_{-0.00200}^{+0.00191}$ & $57140.74073_{-0.00211}^{+0.00204}$ \\
\hline Density $\left(\rho_{\odot}\right)$ & $2.24_{-0.88}^{+0.34}$ & $2.21_{-0.89}^{+0.41}$ & $2.65_{-1.09}^{+0.72}$ & $2.86_{-1.11}^{+0.60}$ & $3.48_{-2.11}^{+1.26}$ & $4.71_{-0.58}^{+0.58}$ & $3.59_{-1.42}^{+0.70}$ \\
\hline Impact Parameter & $0.31_{-0.22}^{+0.29}$ & $0.32_{-0.22}^{+0.29}$ & $0.32_{-0.22}^{+0.30}$ & $0.31_{-0.22}^{+0.28}$ & $0.38_{-0.26}^{+0.37}$ & $1.11_{-0.11}^{+0.11}$ & $0.32_{-0.22}^{+0.29}$ \\
\hline Duration (hr) & $1.61_{-0.02}^{+0.02}$ & $3.32_{-0.09}^{+0.10}$ & $2.68_{-0.13}^{+0.14}$ & $3.47_{-0.12}^{+0.14}$ & $1.44_{-0.09}^{+0.13}$ & $0.57_{-0.03}^{+0.04}$ & $2.64_{-0.08}^{+0.11}$ \\
\hline$a / R_{*}$ & $7.8_{-1.2}^{+0.4}$ & $32.9_{-5.2}^{+1.9}$ & $26.9_{-4.4}^{+2.2}$ & $45.7_{-6.9}^{+3.0}$ & $10.0_{-2.7}^{+1.1}$ & $9.8_{-0.4}^{+0.4}$ & $30.2_{-4.7}^{+1.8}$ \\
\hline Inclination (degrees) & $87.7_{-2.9}^{+1.6}$ & $89.5_{-0.7}^{+0.4}$ & $89.3_{-0.9}^{+0.5}$ & $89.6_{-0.5}^{+0.3}$ & $87.9_{-3.6}^{+1.5}$ & $83.5_{-0.7}^{+0.7}$ & $89.4_{-0.8}^{+0.4}$ \\
\hline$R_{P}{ }^{\mathrm{c}}\left(R_{\oplus}\right)$ & $3.5_{-0.2}^{+0.2}$ & $2.0_{-0.1}^{+0.1}$ & $1.3_{-0.1}^{+0.1}$ & $2.2_{-0.1}^{+0.2}$ & $1.9_{-0.1}^{+0.2}$ & $9.6_{-4.8}^{+5.3}$ & $3.7_{-0.2}^{+0.2}$ \\
\hline \multicolumn{8}{|c|}{ External stellar density prior; uniform priors on $\sqrt{e} \sin \omega, \sqrt{e} \cos \omega$} \\
\hline Period (days) & $1.673916_{-0.000013}^{+0.000012}$ & $14.677286_{-0.000804}^{+0.000828}$ & $9.915615_{-0.001195}^{+0.001209}$ & $21.169619_{-0.001729}^{+0.001665}$ & $1.974190_{-0.000110}^{+0.000110}$ & $\cdots$ & $10.135091_{-0.000488}^{+0.000495}$ \\
\hline$R_{P} / R_{*}$ & $0.0269_{-0.0007}^{+0.0017}$ & $0.0247_{-0.0007}^{+0.0012}$ & $0.0170_{-0.0008}^{+0.0012}$ & $0.0336_{-0.0013}^{+0.0023}$ & $0.0365_{-0.0014}^{+0.0024}$ & $\ldots$ & $0.0771_{-0.0020}^{+0.0033}$ \\
\hline$T_{0}{ }^{\mathrm{b}}(\mathrm{BJD}-2400000)$ & $57144.06723_{-0.00037}^{+0.00055}$ & $57152.68135_{-0.00230}^{+0.00223}$ & $57139.65518_{-0.00552}^{+0.0052}$ & $57123.23803_{-0.00430}^{+0.00443}$ & $57140.38117_{-0.00261}^{+0.00268}$ & $\cdots$ & $57140.74083_{-0.00208}^{+0.00205}$ \\
\hline Density $(\rho \odot)$ & $0.71_{-0.10}^{+0.10}$ & $2.07_{-0.28}^{+0.27}$ & $2.15_{-0.27}^{+0.27}$ & $2.98_{-0.40}^{+0.39}$ & $4.58_{-0.62}^{+0.63}$ & $\cdots$ & $5.03_{-0.72}^{+0.73}$ \\
\hline Impact Parameter & $0.43_{-0.30}^{+0.29}$ & $0.35_{-0.23}^{+0.25}$ & $0.43_{-0.27}^{+0.24}$ & $0.38_{-0.26}^{+0.33}$ & $0.37_{-0.25}^{+0.33}$ & $\cdots$ & $0.32_{-0.22}^{+0.30}$ \\
\hline Duration (hr) & $1.55_{-0.35}^{+0.23}$ & $3.23_{-0.63}^{+0.56}$ & $2.66_{-0.52}^{+0.56}$ & $3.20_{-1.02}^{+0.60}$ & $1.27_{-0.39}^{+0.21}$ & $\ldots$ & $2.28_{-0.49}^{+0.31}$ \\
\hline$a / R_{*}$ & $6.2_{-0.8}^{+0.3}$ & $32.5_{-2.4}^{+2.3}$ & $25.3_{-2.1}^{+1.9}$ & $46.7_{-4.5}^{+9.8}$ & $11.0_{-1.1}^{+2.4}$ & $\cdots$ & $33.2_{-2.7}^{+6.8}$ \\
\hline Inclination (degrees) & $85.1_{-3.1}^{+3.3}$ & $89.4_{-0.5}^{+0.4}$ & $89.0_{-0.6}^{+0.6}$ & $89.5_{-0.4}^{+0.3}$ & $88.0_{-1.9}^{+1.4}$ & $\cdots$ & $89.4_{-0.5}^{+0.4}$ \\
\hline Eccentricity & $0.24_{-0.12}^{+0.19}$ & $0.10_{-0.08}^{+0.18}$ & $0.10_{-0.07}^{+0.16}$ & $0.18_{-0.15}^{+0.27}$ & $0.18_{-0.14}^{+0.29}$ & $\cdots$ & $0.16_{-0.11}^{+0.19}$ \\
\hline$\omega$ (degrees) & $29_{-33}^{+41}$ & $0_{-118}^{+152}$ & $-1_{-132}^{+136}$ & $0_{-67}^{+156}$ & $0_{-63}^{+155}$ & $\ldots$ & $-2_{-52}^{+157}$ \\
\hline$R_{P}{ }^{\mathrm{c}}\left(R_{\oplus}\right)$ & $3.5_{-0.2}^{+0.2}$ & $2.0_{-0.1}^{+0.1}$ & $1.3_{-0.1}^{+0.1}$ & $2.2_{-0.1}^{+0.2}$ & $1.9_{-0.1}^{+0.2}$ & $\ldots$ & $3.7_{-0.2}^{+0.2}$ \\
\hline vespa FPP & $3.6 \times 10^{-3}$ & $1.4 \times 10^{-4}$ & $1.7 \times 10^{-3}$ & $1.9 \times 10^{-4}$ & $7.0 \times 10^{-3}$ & 0.02 & $1.3 \times 10^{-3}$ \\
\hline
\end{tabular}

${ }^{a}$ For EPIC 211901114 the transit duration is unresolved, so we fix $e$ and $\omega$ to zero while simultaneously applying the Gaussian prior on $\rho$. Only one fit is done on this system.

${ }^{b}$ BJD is given in Barycentric Dynamical Time (TBD) format.

${ }^{c}$ Planet radius is derived using our stellar radius from Section 3.

Nоте-Duration, $a / R_{*}$, and inclination are not fit as part of the MCMC; they are calculated from the fit parameters after the run is complete. 